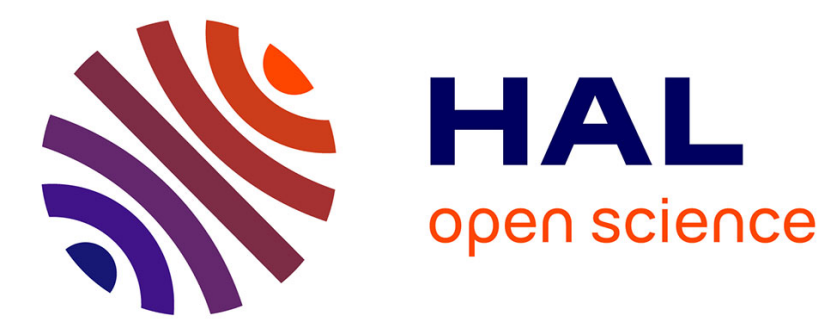

\title{
Gas flow through a bore-piston ring contact
}

\author{
Baptiste Hallouin, Didier Lasseux, Gerald Senger
}

\section{To cite this version:}

Baptiste Hallouin, Didier Lasseux, Gerald Senger. Gas flow through a bore-piston ring contact. International Journal of Engine Research, 2020, pp.146808742097112. 10.1177/1468087420971127 . hal-03041892

\section{HAL Id: hal-03041892 \\ https://hal.science/hal-03041892}

Submitted on 5 Dec 2020

HAL is a multi-disciplinary open access archive for the deposit and dissemination of scientific research documents, whether they are published or not. The documents may come from teaching and research institutions in France or abroad, or from public or private research centers.
L'archive ouverte pluridisciplinaire HAL, est destinée au dépôt et à la diffusion de documents scientifiques de niveau recherche, publiés ou non, émanant des établissements d'enseignement et de recherche français ou étrangers, des laboratoires publics ou privés. 


\title{
Gas flow through a bore-piston ring contact
}

\author{
Baptiste Hallouin ${ }^{1}$, Didier Lasseux ${ }^{2}$ and Gerald Senger ${ }^{1}$
}

\begin{abstract}
This work reports on the derivation of simplified but accurate models to describe gas flow through a bore-piston ring contact, in reciprocating machines like compressors or IC engines. On the basis of the aperture field of a contact deduced from real measurements carried out on an expanding ring in a bore, a scale analysis on the complete compressible flow model is performed, assuming ideal gas law. It is shown that the flow can be treated as stationary and three distinct flow regimes can be identified (namely incompressible, compressible creeping and compressible inertial regimes). Three dimensionless parameters characterizing these regimes are identified. While for the two former regimes, classical analytical Poiseuille type of models are derived, an Oseen approximation is further employed for the latter, yielding a quasi-analytical solution. The models are successfully compared to direct numerical simulations (DNS) of the complete initial set of balance equations in their steady form performed on an aperture field of sinusoidal shape. These simplified models are of particular practical interest since they allow an accurate gas flow-rate estimate through a real contact using the aperture field as the geometrical input datum, together with the thermodynamic conditions (pressure and temperature). This represents an enormous advantage as DNS is hardly tractable still very challenging in practice due to the extremely small value of the contact aperture to contact length ratio.
\end{abstract}

\section{Keywords}

Gas blow-by, bore-piston ring contact, analytical solution

\section{Introduction}

Piston rings are key components of reciprocating machines. Their prime function is to dynamically seal the clearance between the bore and the moving piston in order to maintain gas pressure in the chamber above the piston. However, due to many parameters, among which are technological constraints like open rings as well as geometrical defects that are always present at very different scales on all the contact surfaces (bore, ring and piston groove), significant leak may occur through the piston ring pack. ${ }^{1}$ This represents a major source of pressure loss that may strongly impact the machine efficiency. So far, two main leakage paths have been considered to account for efficiency loss due to gas blow-by. The first one, as the main, corresponds to the ring end gaps. Flow through this leak path is assumed to be one dimensional and isentropic using an "orifice and volume" approach as an equivalence with the flow between two volumes (the two chambers separated by the ring) through an orifice having the gap cross section. ${ }^{2-4}$ The second potential leak path, as reported in some scarce references, can be identified as the clearance between the ring faces and the piston groove flanks as a result of either the absence of contact or only partial contact due to deviations to flatness on both the ring faces and groove flanks. ${ }^{5,6}$ In this region, the flow is approximated by the classical 1D Reynolds equation as was used elsewhere 7,8 and which served as the basis of extensive analyses of contact transmissivities for different classes of surfaces. ${ }^{9-13}$ However, for gas, compressible and inertial effects might be significant as will be further highlighted in the next sections, calling for a more careful investigation of the relevant flow models.
Empirical evidences have shown that piston rings normally experience starved lubrication, at least at some points of their stroke, mainly due to interaction with the other rings in the pack. ${ }^{14,15}$ Simulation methods for the motion of a piston ring pack have been developed, ${ }^{5,6,16-19}$ showing that ring movements affect lubricant consumption and engine wear mechanism. Some of these methods, focused on lubricant starvation issue, have been able to predict the occurrences of oil film rupture and the conditions that can lead to gas blow-by. ${ }^{4}$ The existence of a discontinuous oil film strongly suggests that, in addition to ring end gap and groove flank blow-by, some gas leakage may also appear within the bore-ring interface, i.e. in the gap -referred to as the aperture field in the remainder of this paper- resulting from deviation to circularity of both the ring and bore where oil film is broken up. Experimental observations confirm this assumption and clearly show that the mechanism is enhanced by excessive bore distortions. ${ }^{20,21}$ Nevertheless, the literature dedicated to the analysis of the rings to bore conformity is mainly focused on tribological aspects ${ }^{22-25}$ and the gas flow mechanism through the aperture field has not been enough reported with enough details.

\footnotetext{
${ }^{1}$ Safran Helicopter Engines, Avenue Joseph Szydlowski, 64511 Bordes, France

${ }^{2}$ I2M, UMR 5295, CNRS, Univ. Bordeaux, Esplanade des Arts et Métiers 33405 Talence CEDEX, France
}

\section{Corresponding author:}

Didier Lasseux, I2M, UMR 5295, CNRS, Univ. Bordeaux, Esplanade des Arts et Métiers

33405 Talence CEDEX, France

Email: didier.lasseux@u-bordeaux.fr 
In order to quantify this effect and help the diagnostic and/or the design of a bore-piston assembly, one may think about carrying out direct numerical simulations (DNS) of the complete set of equations (mass, momentum, energy and equation of state) governing the flow. In practice, this is however extremely difficult to routinely perform an accurate computation of the flow through a real aperture field. In fact, the scale contrast between the circumferential extension of the contact (which can be of the order of tens of centimeters, or even one meter in the case of diesel marine engines ${ }^{25}$ ) compared to the characteristic value of the aperture (typically few microns) is such that this requires a tremendously large number of grid blocks, making the simulation intractable for routine use. A reduction of the number of grid points for reasonable computational resources would be to the cost of a mesh featuring grid block with a poor aspect ratio which may significantly alter the accuracy of the results. An alternative would be to employ a multi-scale approach, ${ }^{26}$ which however requires an upscaled model that is extremely difficult to achieve if mass, momentum and energy conservation equations are coupled. Some detailed computation of the gas pressure evolution in combustion chambers was reported recently ${ }^{27}$ but the gas blow-by envisaged here was not taken into account. To circumvent this difficulty, accurate predicting models, requiring very few computational steps, are highly desirable.

Our objective in this work is hence to complement previous descriptions of gas blow-by with predictive models of gas flow through the aperture field of the bore-ring contact that has not been accounted for, so far. In particular, the purpose is to derive simplified models that are of simple practical use that would be able to give a satisfactory approximation of the flow rate through the contact. This is carried out assuming that $i$ ) the gas is ideal; $i i)$ the gas thermophysical parameters (dynamic viscosity, heat capacity at constant pressure and thermal conductivity) are constant; iii) no oil film is present in the contact, or at least, if present at some places on the surfaces, it behaves as a solid with respect to the gas flow; $i v$ )the pressure drop across the ring remains moderate. Within this framework, the article is organized as follows. In section 2 , it is shown that three distinct flow regimes can be identified -namely incompressible, compressible creeping and compressible inertial- that essentially depend on the pressure drop between the ring faces. This is performed from a scale analysis carried out on the full set of balance equations governing the flow. It is shown that the flow can be considered as stationary, the dynamics of the piston being only reflected through the evolution of the velocity of the piston ring outer surface during the stroke. In section 3, operational predictive flowrate models and their solutions are provided for the three identified flow regimes. These simplified predictive models are compared in section 4 to DNS of the flow performed on a model aperture field of realistic characteristic dimensions.

\section{Gas flow model}

The starting point of our analysis is the microscopic description of mass, momentum and heat transfer of the gas within the aperture field, assuming negligible Knudsen effects so that the classical framework of continuum mechanics with no velocity and temperature jumps at the solid boundaries is assumed. The gas is assumed to obey the ideal gas law, and, in addition, thermophysical properties of the gas (dynamic viscosity, $\mu$, thermal conductivity, $\lambda$, and heat capacity at constant pressure, $c_{p}$ ) are assumed to be constant. These assumptions are adopted for the sake of simplicity in the development although the dependence of these parameters on temperature and fuel-to-air ratio may be required in the case of IC engines. From this simplified model, the different flow regimes are identified.

\section{Balance equations}

The flow within the bore-piston ring contact is governed by the classical mass, momentum and energy balance equations which, according to our hypotheses, are recalled below.

* Continuity equation

Mass conservation is that for a classical compressible fluid, i.e.

$$
\frac{\partial \rho}{\partial t}+\rho \nabla \cdot \mathbf{u}+\mathbf{u} \cdot \nabla \rho=0
$$

* Navier-Stokes (momentum) equation

The gas is assumed to be a Newtonian fluid and the two coefficients of viscosity are related by the Stokes relation for which the bulk viscosity is zero. The motion is therefore described by the following momentum equation

$$
\rho\left(\frac{\partial \mathbf{u}}{\partial t}+\mathbf{u} \cdot \nabla \mathbf{u}\right)=-\nabla P+\mu \nabla^{2} \mathbf{u}+\frac{1}{3} \mu \nabla \nabla \cdot \mathbf{u}
$$

* Energy equation

The flow is assumed to be adiabatic and heat conduction in the gas is governed by Fourier's law. Under these assumptions, the energy equation takes the following form

$$
\begin{aligned}
& \frac{\partial}{\partial t}\left(\rho\left(c_{p} T-\frac{P}{\rho}+\frac{u^{2}}{2}\right)\right) \\
& +\rho \mathbf{u} \cdot\left(\frac{1}{2} \nabla u^{2}+c_{p} \nabla T\right)=\nabla \cdot(\boldsymbol{\sigma} \cdot \mathbf{u})+\lambda \nabla^{2} T \\
& \text { * Gas equation of state }
\end{aligned}
$$

The gas behavior is described by the ideal gas state law

$$
P=\rho r T
$$

where $r$ is the ideal gas constant for the gas under consideration. Gas enthalpy, $H$, is related to the temperature by the following relationship

$$
H=\int_{T_{\text {ref }}}^{T} c_{p} d T
$$

The purpose of the scale analysis is to determine the most influential terms in the above equations in order to derive simplified flow models.

\section{Dimensionless form and scale analysis}

The scale analysis is performed on a representative volume of the gas between the bore and the ring, as the one depicted between points A and B in Fig. 1. This figure shows a typical aperture field of a bore-ring interface which was obtained by superimposing real profiles measured on an expanding ring and a bore. Indeed, the aperture field, $h$, resulting from 


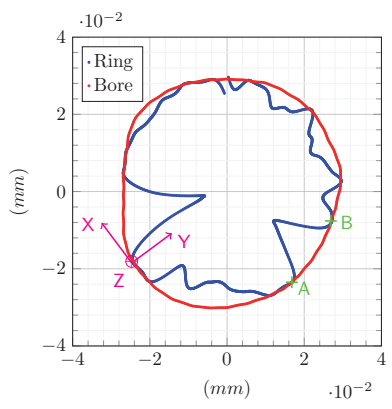

a)

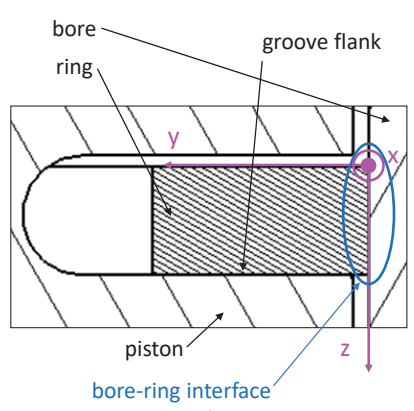

b)
Figure 1. a) Example of a real aperture field resulting from the measurement of ring and bore surfaces. Top view in the $x y$ plane. b) Schematic cross section view of the piston-ring-bore assembly. The ring is in the $x y$ plane.

the difference between the two surfaces in contact, is the geometrical characteristic of interest to analyze the flow.

As indicated by this figure, bore roundness deviation is extremely small since it does not exceed micrometers over the whole circumference. In a bolted assembly, this deviation may be much larger, leading to a loss of conformity between the ring and the bore. Conversely, defects on the ring (resulting from processing, wear, etc.) are much more significant suggesting that they are the main source of the resulting aperture field. Many authors have studied the influence of bore distortions on ring conformability but, most of the time, those on the ring have been disregarded. ${ }^{28-31}$ Few works dedicated to this issue addressed the problem from a tribological point of view. ${ }^{22-25}$ Figure 1 shows that the ring defects should also be taken into account since they can induce an aperture of several hundredths of millimeters. They shall certainly be considered as the main origin of poor conformity.

As the ratio between the typical aperture and the ring radius is much smaller than 1 , the analysis can be carried out using a Cartesian system of coordinates for which $x, y$ and $z$ correspond to the azimuthal, radial and axial directions respectively, the latter being the main direction of the piston motion. Both the upstream and downstream side of the ring are in the $(x, y)$ plane.

Six flow variables are involved in the problem, namely the three components of velocity, u, i.e. $u, v$ and $w$ in the three directions $x, y$ and $z$ respectively, the temperature, $T$, the pressure, $P$, and the density, $\rho$, all being space and time dependent. For a convenient scale analysis, they shall be classified into two categories, depending on whether their scale is defined a priori or not. The term scale for a variable, is meant to be a representative value of the magnitude of its variation. First are the variables whose scale can be easily inferred from the configuration and operating conditions. These are the space variables along with the pressure and time. Scales of space coordinates are determined from the aperture field as represented in Fig. 1. The characteristic dimensions of the aperture field are such that $x$ scales as $L_{0}$, the average distance between two contact points, $y$ scales as $h_{0}$, the typical aperture, $z$ scales as $l_{0}$, the typical width of a ring. Since gas flow through the aperture field is actuated by both the gas pressure gradient across the contact and piston motion, the pressure can be scaled by the pressure drop,
$\Delta P$, between the upstream and downstream faces. Typically, $\triangle P$ is of the order of $10 k P a, 100 k P a$ or $0.1 M P a$, depending on the type of machine and position of the ring in the assembly. It is rather $0.1 M P a$ for the top compression ring of an IC engine, for instance, and typically $100 \mathrm{kPa}$ for a ring in a compressor. The time-scale, $t_{0}$, is determined by the stroke period, i.e.

$$
t_{0}=\frac{c}{V}
$$

where $c$ is the stroke and $V$ is the piston velocity.

The second category of variables includes those whose scale is unknown a priori, which, in the present case, are $u, v, w, T$ and $\rho$. Scales associated to these variables are respectively denoted by $u_{0}, v_{0}, w_{0}, \Delta T$ and $\Delta \rho$. The objective of the scale analysis is first to determine unknown scales and further to eventually simplify the balance equations by discarding unimportant terms on the basis of their respective orders of magnitude. To do so, unknown scales have to be expressed as functions of known scales and this is achieved once all variables and their derivatives are made dimensionless and are all of order one according to their respective scales. From a practical point of view, this means that all the variables are bounded between roughly 0.1 and 10 . The comparison between the different terms in the equations can be then performed from the order of magnitude of their respective prefactors only. Prefactors have thus to be expressed as functions of known scales so that they can be quantified numerically.

To have dimensionless variables of order 1 , two changes of variables may be performed. The first one is purely linear. It is suitable for variables whose variation and average value have the same orders of magnitude. In particular, it is convenient for all the variables that are set to zero at some location at a given time. This is the case for velocity components as no slip is assumed at the gas-solid interface. This is also the case for space and time coordinates as a result of the choice of the Cartesian triad and time origins. This leads to the following changes of variables

$$
\begin{gathered}
x^{*}=\frac{x}{L_{0}}, y^{*}=\frac{y}{h_{0}}, z^{*}=\frac{z}{l_{0}} \\
u^{*}=\frac{u}{u_{0}}, v^{*}=\frac{v}{v_{0}}, w^{*}=\frac{w}{w_{0}} \\
t^{*}=\frac{t}{t_{0}}
\end{gathered}
$$

Conversely, variables whose variation is small compared to their average value require an affine change of variable. It shall be noted that, in the absence of any indication on whether the variable itself experiences small variations compared to its average value, an affine change of variable is preferable to a linear one, even though the analysis might be more complex. In our case, pressure, temperature and gas density are respectively made dimensionless as follows

$$
\begin{aligned}
P^{*} & =\frac{P-P_{0}}{\Delta P} \\
T^{*} & =\frac{T-T_{0}}{\Delta T} \\
\rho^{*} & =\frac{\rho-\rho_{0}}{\Delta \rho}
\end{aligned}
$$


In these three last relationships, $P_{0}, T_{0}$ and $\rho_{0}$ are reference values which can be taken as those at a boundary. For instance, $P_{0}$ can be chosen as the gas pressure in the crankcase in the case of an IC engine.

The governing equations (1) to (4) are rewritten according to the different changes of variables in equations (7) and (8) and unknown scales are determined by matching terms involving them, i.e. by solving the equations in terms of the orders of magnitude, according to a least degeneracy principle. This is a classical approach in the method of matched asymptotic expansions. ${ }^{32}$ With this, it is simply meant that the unknown scales must be determined so that the resulting number of negligible terms in the balance equations of the problem is minimal. The scale analysis is detailed in the Appendix in which a special attention is dedicated to the temperature field and this leads to the following solution

$$
\begin{aligned}
& u_{0} \sim \frac{h_{0}^{2} \Delta P}{\mu L_{0}} \\
& v_{0} \sim \frac{h_{0}^{3} \Delta P}{\mu l_{0}^{2}} \\
& w_{0} \sim \frac{h_{0}^{2} \Delta P}{\mu l_{0}} \\
& \Delta T \sim \frac{h_{0}^{4} \Delta P^{2}}{\mu l_{0}^{2} \lambda} \\
& \Delta \rho \sim \rho_{0}\left(\frac{1+\tau}{1+\operatorname{Re} \delta \tau \frac{\mu r}{\lambda}}-1\right)
\end{aligned}
$$

In this last relationship, $\tau$ and $R e$ are respectively given by

$$
\begin{gathered}
\tau=\frac{\Delta P}{P_{0}} \\
\operatorname{Re}=\frac{\rho_{0} h_{0} w_{0}}{\mu}=\frac{P_{0} h_{0}^{3} \Delta P}{r T_{0} \mu^{2} l_{0}}
\end{gathered}
$$

where $R e$ is the Reynolds number of the flow. Taking into account the range of $\Delta P$ mentioned above, $\tau$ is considered to have an order of magnitude up to 1 in the following.

With this solution in terms of orders of magnitude at hand, the dimensionless governing equations can be written as

* Continuity equation

$$
\begin{aligned}
& \frac{\alpha}{\tau}\left(\frac{1}{\frac{1}{\tau}+P^{*}} \frac{\partial P^{*}}{\partial t^{*}}-\frac{1}{\frac{1}{\operatorname{Re} \tau} \frac{\lambda}{\mu r}+T^{*}} \frac{\partial T^{*}}{\partial t^{*}}\right) \\
& +\frac{\partial v^{*}}{\partial y^{*}}+\frac{\partial w^{*}}{\partial z^{*}} \\
& +v^{*}\left(\frac{1}{\frac{1}{\tau}+P^{*}} \frac{\partial P^{*}}{\partial y^{*}}-\frac{1}{\frac{1}{\operatorname{Re} \tau} \frac{\lambda}{\mu r}+T^{*}} \frac{\partial T^{*}}{\partial y^{*}}\right) \\
& +w^{*}\left(\frac{1}{\frac{1}{\tau}+P^{*}} \frac{\partial P^{*}}{\partial z^{*}}-\frac{1}{\frac{1}{\operatorname{Re} \tau} \frac{\lambda}{\mu r}+T^{*}} \frac{\partial T^{*}}{\partial z^{*}}\right) \\
& =O\left((\epsilon / \delta)^{2}\right)
\end{aligned}
$$

* Navier-Stokes equation

$$
\begin{aligned}
& \frac{1+\tau P^{*}}{1+\operatorname{Re} \delta \frac{\mu r}{\lambda} \tau T^{*}} \\
& \operatorname{Re} \delta\left(\frac{\alpha}{\tau} \frac{\partial u^{*}}{\partial t^{*}}+v^{*} \frac{\partial u^{*}}{\partial y^{*}}+w^{*} \frac{\partial u^{*}}{\partial z^{*}}+O\left((\epsilon / \delta)^{2}\right)\right) \\
& =-\frac{\partial P^{*}}{\partial x^{*}}+\frac{\partial^{2} u^{*}}{\partial y^{* 2}}+O\left(\epsilon^{2}\right)+O\left(\delta^{2}\right)
\end{aligned}
$$

$$
\begin{aligned}
& \frac{1+\tau P^{*}}{1+\operatorname{Re} \delta \frac{\mu r}{\lambda} \tau T^{*}} \\
& \operatorname{Re}^{3}\left(\frac{\alpha}{\tau} \frac{\partial v^{*}}{\partial t^{*}}+v^{*} \frac{\partial v^{*}}{\partial y^{*}}+w^{*} \frac{\partial v^{*}}{\partial z^{*}}+O\left((\epsilon / \delta)^{2}\right)\right) \\
& =-\frac{\partial P^{*}}{\partial y^{*}}+O\left(\epsilon^{2}\right)+O\left(\delta^{2}\right)
\end{aligned}
$$

$$
\begin{aligned}
& \frac{1+\tau P^{*}}{1+\operatorname{Re} \delta \frac{\mu r}{\lambda} \tau T^{*}} \\
& \operatorname{Re} \delta\left(\frac{\alpha}{\tau} \frac{\partial w^{*}}{\partial t^{*}}+v^{*} \frac{\partial w^{*}}{\partial y^{*}}+w^{*} \frac{\partial w^{*}}{\partial z^{*}}+O\left((\epsilon / \delta)^{2}\right)\right) \\
& =-\frac{\partial P^{*}}{\partial z^{*}}+\frac{\partial^{2} w^{*}}{\partial y^{* 2}}+O\left(\epsilon^{2}\right)+O\left(\delta^{2}\right)
\end{aligned}
$$

* Energy equation

$$
\begin{aligned}
& \frac{\alpha}{\tau} \operatorname{Re} \delta\left\{\left[\left(\operatorname{Pr}-\frac{\mu r}{\lambda}\right)\left(1+\operatorname{Re} \delta \tau \frac{\mu r}{\lambda} T^{*}\right)\right.\right. \\
& \left.+\frac{1}{2}\left(O\left((\epsilon / \delta)^{2}\right)+O\left(\delta^{2}\right)+w^{* 2}\right)\right] \\
& \left(\frac{1+\tau}{1+\operatorname{Re} \delta \tau \frac{\mu r}{\lambda}}-1\right) \frac{\partial \rho^{*}}{\partial t^{*}} \\
& +\left(1+\left(\frac{1+\tau}{1+\operatorname{Re} \delta \tau \frac{\mu r}{\lambda}}-1\right) \rho^{*}\right)
\end{aligned}
$$$$
\left.\left(\left(P r-\frac{\mu r}{\lambda}\right) \frac{\partial T^{*}}{\partial t^{*}}+O\left((\epsilon / \delta)^{2}\right)+O\left(\delta^{2}\right)+w^{*} \frac{\partial w^{*}}{\partial t^{*}}\right)\right\}
$$$$
+\frac{1+\tau P^{*}}{1+\operatorname{Re} \delta \tau \frac{\mu r}{\lambda} T^{*}} \operatorname{Re} \delta\left(w^{*}\left(v^{*} \frac{\partial w^{*}}{\partial y^{*}}+w^{*} \frac{\partial w^{*}}{\partial z^{*}}\right)\right.
$$$$
\left.+\operatorname{Pr}\left(v^{*} \frac{\partial T^{*}}{\partial y^{*}}+w^{*} \frac{\partial T^{*}}{\partial z^{*}}\right)\right)
$$$$
=\frac{\partial^{2} T^{*}}{\partial y^{* 2}}+\frac{\partial}{\partial y^{*}}\left(w^{*} \frac{\partial w^{*}}{\partial y^{*}}\right)+O\left((\epsilon / \delta)^{2}\right)
$$$$
+O\left(\epsilon^{2}\right)+O\left(\delta^{2}\right)
$$

* Gas equation of state

$$
\begin{aligned}
1+\tau P^{*}= & \left(1+\left(\frac{1+\tau}{1+\operatorname{Re} \delta \tau \frac{\mu r}{\lambda}}-1\right) \rho^{*}\right) \\
& \left(1+\operatorname{Re} \delta \tau \frac{\mu r}{\lambda} T^{*}\right)
\end{aligned}
$$

In these equations, the following nomenclature was employed

$$
\begin{gathered}
\delta=\frac{h_{0}}{l_{0}} \\
\epsilon=\frac{h_{0}}{L_{0}} \\
\alpha=\frac{\mu V l_{0}^{2}}{P_{0} c h_{0}^{2}}
\end{gathered}
$$

and

$$
\operatorname{Pr}=\frac{\mu c_{p}}{\lambda}
$$

where $\operatorname{Pr}$ is the Prandtl number of the flow. Since $\operatorname{Pr}$ and $\frac{\mu r}{\lambda}$ are both of order 1 , the flow within the bore-ring interface 
is conditioned by $\tau$ and two $\tau$-dependent dimensionless numbers, namely $\frac{\alpha}{\tau}$ and $\operatorname{Re} \delta$ with

$$
\operatorname{Re} \delta=\beta \tau
$$

and

$$
\beta=\frac{P_{0}^{2}}{r T_{0}} \frac{h_{0}^{4}}{\mu^{2} l_{0}^{2}}
$$

The dimensionless momentum equations clearly show that the balance between inertial and viscous effects does not simply depend on the Reynolds number but on the rescaled form, $R e \delta$, of this number.

Characteristic dimensions of realistic aperture fields are such that the ratios $\delta$ and $\epsilon$ are both small compared to unity. In fact, taking typical values as $h_{0} \sim 10^{-5} \mathrm{~m}, l_{0}=$ $10^{-3} \mathrm{~m}$ and $L_{0}=10^{-2} \mathrm{~m}$ leads to $\delta \sim 10^{-2}$ and $\epsilon \sim$ $10^{-3}$. As a consequence, the governing equations (1) to (4) can be simplified on the basis of comparative orders of magnitude of the different terms in these equations depending on the order of magnitude of $\tau$ and this is carried out in the following section, with the purpose of determining a simplified solution for the axial component, $w$, of the velocity in order to estimate the gas blow-by through the bore-piston ring contact.

\section{Flow regimes identification}

The flow regime within the bore-ring interface depends on the order of magnitude of $\operatorname{Re} \delta=\beta \tau$, i.e. on $\tau$ which is intimately related to the type of reciprocating machine under consideration as well as to the location of the ring in the ring pack. It is also linked to the value of $\beta$ which depends on the bore-ring conformity. As discussed above, the aperture field for this contact can take typical values of several hundredths of millimeters. However, in some cases of better conformity, occurring in the wear regime for example, it decreases to few micrometers.

To begin with, the influence of the non-stationary terms in the mass, momentum and energy balance equations (12), (13) and (14) respectively must be investigated. A careful inspection indicates that the importance of these terms relies on the prefactors $\alpha$ in the mass equation and $\alpha \beta$ in both the momentum and energy equations. As a consequence, typical values of the two parameters $\alpha$ and $\beta$ must be estimated. For such an estimation, the following characteristic orders of magnitude, representative of realistic situations, are used: $P_{0} \sim 10^{5} \mathrm{~Pa}, V \sim 10 \mathrm{~ms}^{-1}, \mathrm{c} \sim 10^{-1} \mathrm{~m}, \mathrm{~T}_{0} \sim 300 \mathrm{~K}$. It should be noted that $T_{0}$ is taken as the ambient temperature with an order of magnitude intermediate between $10^{2}$ and $10^{3}$. For $h_{0}$, one may distinguish situations where conformity between the ring and the bore is either poor or of good quality. In the former case, it is reasonable to consider $h_{0} \sim$ $10^{-5} \mathrm{~m}$, while in the latter, one may rather take $h_{0} \sim 10^{-6}$ $m$. This respectively yields

$$
\begin{gathered}
\alpha=\frac{\mu V l_{0}^{2}}{P_{0} c h_{0}^{2}} \sim 10^{-4} \\
\beta=\frac{P_{0}^{2}}{r T_{0}} \frac{h_{0}^{4}}{\mu^{2} l_{0}^{2}} \sim 1
\end{gathered}
$$

when $h_{0} \sim 10^{-5} m$ and

$$
\alpha \sim 10^{-2}
$$

$$
\beta \sim 10^{-4}
$$

when $h_{0} \sim 10^{-6} \mathrm{~m}$. In the two situations, $\alpha$ and $\alpha \beta$ are much smaller than 1 , and this indicates that unsteady terms in the mass, momentum and energy equations are completely negligible, showing that non-stationary effects induced by the piston movement on the gas flow through the aperture field can be neglected.

With this simplification at hand, the flow regimes can now be analyzed and this is performed in accordance with the orders of magnitude of $\tau$ and $\beta$. Keeping in mind that $\beta$ can be up to $O(1)$, the discussion is mainly focused on $\tau$. The purpose is to derive approximate models which can yield simple solutions that can be carried out analytically as much as possible.

Incompressible flow regime This regime occurs when $\tau \ll$ 1 . The corresponding model is hence obtained using a zerothorder approximation in $\tau$ in equations (12) to (15) above which can be expressed as

* Continuity equation

$$
O(\alpha)+\frac{\partial v^{*}}{\partial y^{*}}+\frac{\partial w^{*}}{\partial z^{*}}+O(\tau)+O\left((\epsilon / \delta)^{2}\right)=0
$$

* Navier-Stokes equations

$$
\begin{gathered}
O(\alpha \beta)+O(\tau)=-\frac{\partial P^{*}}{\partial x^{*}}+\frac{\partial^{2} u^{*}}{\partial y^{* 2}} \\
+O\left(\epsilon^{2}\right)+O\left(\delta^{2}\right) \\
\delta^{2}(O(\alpha \beta)+O(\tau))=-\frac{\partial P^{*}}{\partial y^{*}}+O\left(\epsilon^{2}\right)+O\left(\delta^{2}\right) \\
O(\alpha \beta)+O(\tau)=-\frac{\partial P^{*}}{\partial z^{*}}+\frac{\partial^{2} w^{*}}{\partial y^{* 2}} \\
+O\left(\epsilon^{2}\right)+O\left(\delta^{2}\right)
\end{gathered}
$$

* Energy equation

$$
\begin{aligned}
& O(\alpha \beta \tau)+\frac{\partial^{2} T^{*}}{\partial y^{* 2}}+\frac{\partial}{\partial y^{*}}\left(w^{*} \frac{\partial w^{*}}{\partial y^{*}}\right) \\
& =O\left((\epsilon / \delta)^{2}\right)+O\left(\epsilon^{2}\right)+O\left(\delta^{2}\right)
\end{aligned}
$$

* Gas equation of state

$$
P^{*} O(\tau)=\rho^{*} O(\tau)
$$

The flow is actually incompressible, as shown by equation (24), and in the creeping regime, as indicated by equations (22). The momentum equations can then be integrated. To carry out the integration, the following boundary conditions shall be considered

$$
\begin{gathered}
P(z=0)=P_{1}(x) \\
P(z=l)=P_{0} \\
w(y=0)=0 \\
w(y=h)=V
\end{gathered}
$$


the latter corresponding to a no slip condition at the gasring interface. With such conditions, the integration leads to a solution similar to a Couette flow given by

$$
\begin{gathered}
P(x, z)=\frac{P_{0}-P_{1}(x)}{l} z+P_{1}(x) \\
u(x, y, z)=\frac{1}{2 \mu} \frac{\partial P}{\partial x}\left(y^{2}-h(x) y\right) \\
v=0 \\
w(x, y)=\frac{P_{0}-P_{1}(x)}{2 \mu l}\left(y^{2}-h(x) y\right)+\frac{V}{h(x)} y
\end{gathered}
$$

Compressible creeping flow regime When $\tau$ increases but remains smaller than 1 , compressibility effects become more significant and can be taken into account by a first order approximation in $\tau$ in equations (12) to (15). Doing so yields the following problem statement

* Continuity equation

$$
\begin{aligned}
& O(\alpha)+\frac{\partial v^{*}}{\partial y^{*}}+\frac{\partial w^{*}}{\partial z^{*}}+v^{*}\left(\tau \frac{\partial P^{*}}{\partial y^{*}}+O\left(\tau^{2}\right)\right) \\
& +w^{*}\left(\tau \frac{\partial P^{*}}{\partial z^{*}}+O\left(\tau^{2}\right)\right)+O\left((\epsilon / \delta)^{2}\right)=0
\end{aligned}
$$

* Navier-Stokes equations

$$
\begin{gathered}
O(\alpha \beta)+\beta \tau\left(v^{*} \frac{\partial u^{*}}{\partial y^{*}}+w^{*} \frac{\partial u^{*}}{\partial z^{*}}+O\left((\epsilon / \delta)^{2}\right)\right)+O\left(\tau^{2}\right) \\
=-\frac{\partial P^{*}}{\partial x^{*}}+\frac{\partial^{2} u^{*}}{\partial y^{* 2}}+O\left(\epsilon^{2}\right)+O\left(\delta^{2}\right) \\
\delta^{2}\left[O(\alpha \beta)+\beta \tau\left(v^{*} \frac{\partial v^{*}}{\partial y^{*}}+w^{*} \frac{\partial v^{*}}{\partial z^{*}}+O\left((\epsilon / \delta)^{2}\right)\right)+\right. \\
\left.O\left(\tau^{2}\right)\right]=-\frac{\partial P^{*}}{\partial y^{*}}+O\left(\epsilon^{2}\right)+O\left(\delta^{2}\right) \\
O(\alpha \beta)+\beta \tau\left(v^{*} \frac{\partial w^{*}}{\partial y^{*}}+w^{*} \frac{\partial w^{*}}{\partial z^{*}}+O\left((\epsilon / \delta)^{2}\right)\right) \\
+O\left(\tau^{2}\right)=-\frac{\partial P^{*}}{\partial z^{*}}+\frac{\partial^{2} w^{*}}{\partial y^{* 2}}+O\left(\epsilon^{2}\right)+O\left(\delta^{2}\right) \quad(31 \mathrm{c})
\end{gathered}
$$

* Energy equation

$$
\begin{aligned}
& O(\alpha \beta \tau)+\beta \tau(1+O(\tau))\left[w^{*}\left(v^{*} \frac{\partial w^{*}}{\partial y^{*}}+w^{*} \frac{\partial w^{*}}{\partial z^{*}}\right)\right. \\
& \left.+\operatorname{Pr}\left(v^{*} \frac{\partial T^{*}}{\partial y^{*}}+w^{*} \frac{\partial T^{*}}{\partial z^{*}}\right)\right] \\
& =\frac{\partial^{2} T^{*}}{\partial y^{* 2}}+\frac{\partial}{\partial y^{*}}\left(w^{*} \frac{\partial w^{*}}{\partial y^{*}}\right) \\
& +O\left((\epsilon / \delta)^{2}\right)+O\left(\epsilon^{2}\right)+O\left(\delta^{2}\right)
\end{aligned}
$$

* Gas equation of state

$$
1+\tau P^{*}=1+\tau \rho^{*}+O\left(\beta \tau^{2}\right)
$$

If $\beta$ is of order 1 , (i.e. in the case of poor conformity of the ring in the bore, see equation (20b)), the flow is inertial and an analytical solution of the above equations cannot be found. If $\beta<1$, (i.e. in the case of a good conformity between the bore and the ring, see equation (20d)), the flow remains in the creeping regime as can be inferred from equations (31). Using the boundary conditions given in equations (25) to (28), the integration of the flow equations can be performed yielding the following solution

$$
\begin{gathered}
P(x, z)=\left(\frac{P_{0}^{2}-P_{1}^{2}(x)}{l} z+P_{1}^{2}(x)\right)^{1 / 2} \\
u(x, y, z)=\frac{1}{2 \mu} \frac{\partial P}{\partial x}\left(y^{2}-h(x) y\right) \\
v=0 \\
w(x, y, z)=\frac{\left(P_{0}^{2}-P_{1}^{2}(x)\right)\left(y^{2}-h(x) y\right)}{4 \mu l\left(\frac{P_{0}^{2}-P_{1}^{2}(x)}{l} z+P_{1}^{2}(x)\right)^{1 / 2}} \\
+\frac{V}{h(x)} y
\end{gathered}
$$

Unlike the case of the incompressible regime, it shall be noted that the axial pressure variation is non linear and fluid particles are accelerated in the axial direction.

Compressible inertial flow regime In this case for which $\tau$ is of order 1, inertial (i.e., convective acceleration in the gas) and thermal effects remain significant which is in contrast with situations for which $\tau<1$. Once unsteady terms are discarded and taking into account the values of $\delta$ and $\epsilon$ that are both small compared to 1 , the governing equations can be expressed as

* Continuity equation

$$
\begin{aligned}
& O(\alpha)+\frac{\partial v^{*}}{\partial y^{*}}+\frac{\partial w^{*}}{\partial z^{*}} \\
& +v^{*}\left(\frac{1}{\frac{1}{\tau}+P^{*}} \frac{\partial P^{*}}{\partial y^{*}}-\frac{1}{\frac{1}{\operatorname{Re} \tau} \frac{\lambda}{\mu r}+T^{*}} \frac{\partial T^{*}}{\partial y^{*}}\right) \\
& +w^{*}\left(\frac{1}{\frac{1}{\tau}+P^{*}} \frac{\partial P^{*}}{\partial z^{*}}-\frac{1}{\frac{1}{\operatorname{Re} \tau} \frac{\lambda}{\mu r}+T^{*}} \frac{\partial T^{*}}{\partial z^{*}}\right) \\
& +O\left((\epsilon / \delta)^{2}\right)=0
\end{aligned}
$$

* Navier-Stokes equation

$$
\begin{gathered}
O(\alpha)+\frac{1+\tau P^{*}}{1+\operatorname{Re} \delta \frac{\mu r}{\lambda} \tau T^{*}} \operatorname{Re} \delta \\
\left(v^{*} \frac{\partial u^{*}}{\partial y^{*}}+w^{*} \frac{\partial u^{*}}{\partial z^{*}}+O\left((\epsilon / \delta)^{2}\right)\right) \\
=-\frac{\partial P^{*}}{\partial x^{*}}+\frac{\partial^{2} u^{*}}{\partial y^{* 2}}+O\left(\epsilon^{2}\right)+O\left(\delta^{2}\right) \\
\delta^{2} O(\alpha)=-\frac{\partial P^{*}}{\partial y^{*}}+O\left(\epsilon^{2}\right)+O\left(\delta^{2}\right) \\
O(\alpha)+\frac{1+\tau P^{*}}{1+\operatorname{Re} \delta \frac{\mu r}{\lambda} \tau T^{*}} \operatorname{Re} \delta \\
\left(v^{*} \frac{\partial w^{*}}{\partial y^{*}}+w^{*} \frac{\partial w^{*}}{\partial z^{*}}+O\left((\epsilon / \delta)^{2}\right)\right) \\
=-\frac{\partial P^{*}}{\partial z^{*}}+\frac{\partial^{2} w^{*}}{\partial y^{* 2}}+O\left(\epsilon^{2}\right)+O\left(\delta^{2}\right)
\end{gathered}
$$


* Energy equation

$$
\begin{aligned}
& O(\alpha)+\frac{1+\tau P^{*}}{1+\operatorname{Re} \delta \tau \frac{\mu r}{\lambda} T^{*}} \operatorname{Re} \delta \\
& {\left[w^{*}\left(v^{*} \frac{\partial w^{*}}{\partial y^{*}}+w^{*} \frac{\partial w^{*}}{\partial z^{*}}\right)\right.} \\
& \left.+\operatorname{Pr}\left(v^{*} \frac{\partial T^{*}}{\partial y^{*}}+w^{*} \frac{\partial T^{*}}{\partial z^{*}}\right)\right] \\
& =\frac{\partial^{2} T^{*}}{\partial y^{* 2}}+\frac{\partial}{\partial y^{*}}\left(w^{*} \frac{\partial w^{*}}{\partial y^{*}}\right) \\
& +O\left((\epsilon / \delta)^{2}\right)+O\left(\epsilon^{2}\right)+O\left(\delta^{2}\right)
\end{aligned}
$$

* Gas equation of state

$$
\begin{aligned}
& 1+\tau P^{*}=\left(1+\left(\frac{1+\tau}{1+\operatorname{Re} \delta \tau \frac{\mu r}{\lambda}}-1\right) \rho^{*}\right) \\
& \left(1+\operatorname{Re} \delta \tau \frac{\mu r}{\lambda} T^{*}\right)
\end{aligned}
$$

Under this form, no analytical solution can be obtained. However, some additional simplification may be proposed to carry out a solution of practical use and this is detailed in the following section.

Before proceeding, it should be noted that this last regime can be extended without any modification to the case $\operatorname{Re} \delta>1$ (i.e. $\tau>1$ ) that may be of interest for IC engines for instance, for which, in particular, a good borering conformity is expected.

\section{Simplified operational models}

In this section, simplified models are proposed in order to predict the flow rate through the bore-ring interface in the three flow regimes identified above. This is achieved by deriving the corresponding quasi-analytical solution for the mass flow rate in the axial direction.

\section{Incompressible flow model $(\tau \ll 1)$}

The pressure and velocity fields are given by the analytical solution reported in equations (29), and, to complete this solution, the static pressure $P_{1}(x)$ must be made explicit by setting boundary conditions at the upstream and downstream edges of the bore-ring contact. Realistic boundary conditions are chosen to be such that:

- the static pressure $P_{0}$ is uniform at the downstream face of bore-ring contact, i.e. at the plane $z=l$;

- the total pressure is uniform at the upstream face of the bore-ring contact, i.e. at the plane $z=0$. This can be expressed as

$$
P_{t 1}=P_{1}(x)\left(1+\frac{\gamma-1}{2} \bar{M}_{1}^{2}(x)\right)^{\frac{\gamma}{\gamma-1}}
$$

with

$$
\overline{M_{1}}(x)=\frac{\overline{w_{1}}(x)}{\sqrt{\gamma r T_{1}(x)}}
$$

$\overline{w_{1}}(x)$ being the average axial velocity component at the upstream face of the bore-ring contact

$$
\begin{aligned}
\overline{w_{1}}(x) & =\frac{1}{h} \int_{0}^{h} w_{1}(x, y, z=0) d y \\
& =\frac{P_{1}(x)-P_{0}}{12 \mu l} h^{2}(x)+\frac{V}{2}
\end{aligned}
$$

- the total temperature is uniform at the upstream face of the bore-ring contact. This yields

$$
T_{t 1}=T_{1}(x)\left(1+\frac{\gamma-1}{2} \bar{M}_{1}^{2}(x)\right)
$$

To estimate the blow-by through the bore-ring contact, the aperture field $h(x)$ is discretized according to a finite difference type of discretization using $n$ grid points. For each grid block positioned at $x_{i}, P_{1}, \overline{w_{1}}$ and $T_{1}$ can be computed by solving numerically the set of equations (39) to (42). The flow rate per unit length can finally be obtained at the corresponding position $x_{i}$. It is given by

$$
\begin{aligned}
\frac{d Q_{m}}{d x}\left(x_{i}\right)=\frac{P_{1}\left(x_{i}\right)}{r T_{1}\left(x_{i}\right)} & {\left[\frac{P_{1}\left(x_{i}\right)-P_{0}}{12 \mu l} h^{3}\left(x_{i}\right)\right.} \\
& \left.+\frac{V h\left(x_{i}\right)}{2}\right]
\end{aligned}
$$

The blow-by through the contact is finally obtained as

$$
Q_{m}=\sum_{i=1}^{n} d Q_{m}\left(x_{i}\right)
$$

\section{Compressible creeping flow model $(\tau<1)$}

This simplified model is similar to the incompressible creeping flow regime as the boundary conditions and the procedure to obtain the mass flow rate are the same. Using the expressions of the pressure and velocities given by the analytical solution in equations (34) and employing the methodology presented above, the following expression of the average axial velocity at the upstream face of the borering contact is obtained

$$
\begin{aligned}
\overline{w_{1}}(x) & =\frac{1}{h(x)} \int_{0}^{h} w_{1}(x, y, z=0) d y \\
& =\frac{P_{1}^{2}(x)-P_{0}^{2}}{24 \mu l P_{1}(x)} h^{2}(x)+\frac{V}{2}
\end{aligned}
$$

From this expression, the mass flow rate per unit length through the bore-ring contact is given by

$$
\begin{aligned}
\frac{d Q_{m}}{d x}\left(x_{i}\right)=\frac{P_{1}\left(x_{i}\right)}{r T_{1}\left(x_{i}\right)} & {\left[\frac{P_{1}^{2}\left(x_{i}\right)-P_{0}^{2}}{24 \mu l P_{1}\left(x_{i}\right)} h^{3}\left(x_{i}\right)\right.} \\
& \left.+\frac{V h\left(x_{i}\right)}{2}\right]
\end{aligned}
$$

\section{Compressible inertial flow model $(\tau=O(1))$}

In this case, the set of PDEs governing the flow cannot be simply integrated analytically. For an analytical solution to be tractable, some additional simplifying assumptions are required which consist in assuming that $i$ ) temperature variations remain small within the contact so that the flow 
can be considered as quasi-isothermal, $i$ ) the radial velocity component, $v$, is much smaller than the axial component and can be neglected in the problem.

Under these assumptions equations (35) and (36c) can be combined yielding the approximated equation of motion, which in its dimensional form, is given by

$$
\frac{w^{2}}{r T} \frac{\partial P}{\partial z}=-\frac{\partial P}{\partial z}+\mu \frac{\partial^{2} w}{\partial y^{2}}
$$

This equation remains non linear and to achieve integration, an Oseen approximation shall be used which consists in linearizing the term $\frac{w^{2}}{r T} \frac{\partial P}{\partial z}$. To do so, this term is approximated by $\frac{\bar{w}^{2}}{r T} \frac{\partial P}{\partial z}$ where $\bar{w}(x)$ is the average velocity axial component given by

$$
\bar{w}(x)=\frac{1}{l} \int_{0}^{l}\left(\frac{1}{h(x)} \int_{0}^{h(x)} w(x, y, z) d y\right) d z
$$

The final integration of equation (47) leads to the following solution

$$
\begin{aligned}
P(x, z)= & \left(\frac{P_{0}^{2}-P_{1}^{2}(x)}{l} z+P_{1}^{2}(x)\right)^{1 / 2} \\
w(x, y, z)= & \frac{K\left(P_{0}^{2}-P_{1}^{2}(x)\right)\left(y^{2}-h(x) y\right)}{4 \mu l\left(\frac{P_{0}^{2}-P_{1}^{2}(x)}{l} z+P_{1}^{2}(x)\right)^{1 / 2}} \\
& +\frac{V}{h(x)} y
\end{aligned}
$$

with

$$
K=1-\frac{\bar{w}^{2}(x)}{r T}
$$

and

$$
\bar{w}(x)=\frac{h^{2}(x)}{12 \mu l}\left(1-\frac{\bar{w}^{2}(x)}{r T_{1}(x)}\right)\left(P_{1}(x)-P_{0}\right)+\frac{V}{2}
$$

The same boundary conditions as those employed so far (uniform static pressure, $P_{0}$, at the downstream face and uniform total pressure, $P_{t 1}$, and total temperature, $T_{t 1}$, at the downstream face of the bore-ring contact; see equations (39) and (42)) shall now be considered. This allows to express the average axial velocity component at the upstream face of the bore-ring contact as

$$
\begin{aligned}
\overline{w_{1}}(x) & =\frac{1}{h(x)} \int_{0}^{h(x)} w_{1}(x, y, z=0) d y \\
& =\left(1-\frac{\bar{w}^{2}(x)}{r T_{1}(x)}\right) \frac{P_{1}^{2}(x)-P_{0}^{2}}{24 \mu l P_{1}(x)} h^{2}(x)+\frac{V}{2}
\end{aligned}
$$

The mass flow-rate through the bore-ring contact is finally obtained using the same procedure as the one employed in the two preceding cases.

It should be emphasized that the flow rates computation from the quasi analytical procedures detailed above for each flow regime only requires few seconds, compared to the computational time of a complete DNS, which is typically on the order of 1 to 10 hours.

\section{Comparison with DNS}

In this section, the validity of the simplified models derived above is assessed by comparing their predictions to the results obtained from DNS of the complete initial set of equations. This is performed on a model contact which dimensional characteristics are close to a real configuration. It should be noted that in a real configuration, the data required to employ the predictive models reduce to the aperture field, $h$, resulting from the difference between the external ring and internal bore surfaces as illustrated in Fig. 1.

\section{Configuration}

The geometry of the numerical model used for the comparison between the predictive models and the DNS is a small volume of fluid whose ratios $\epsilon$ and $\delta$ are the same as those of a real aperture field. It is made of a sinusoidal aperture field (see Fig. 2) which varies from 3 to 20 micrometers. Its extent is 18 millimeters in the azimuthal direction and 1 millimeter in the axial direction.

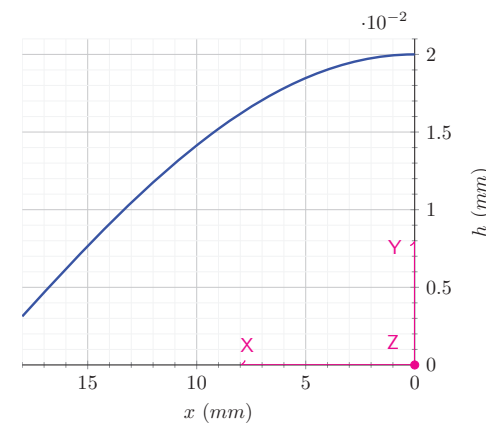

a)

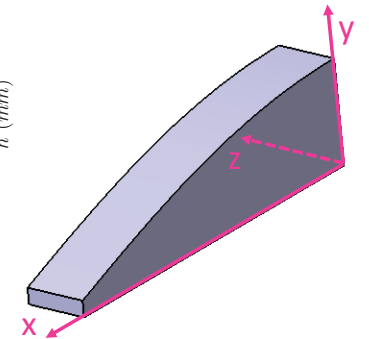

b)
Figure 2. Geometry of the numerical model. a) Aperture field from which the volume of fluid is built. b) Volume of fluid.

The fluid domain was discretized using a quadrilateral mesh made of about 215000 nodes, namely 21 in the radial direction, 51 in the axial direction and 201 in the azimuthal direction.

Equations (1) to (3) in their steady version, together with equation (4), are employed for the DNS with the purpose of analyzing the impact of $\tau$ ( $\beta$ being of $O(1)$ ). The boundary conditions, as detailed in Fig. 3, are those employed to derive the simplified models except that the total temperature is assumed to be uniform on the downstream face of the borering contact. This is a consequence of the fact the hypothesis of an isothermal flow is not kept in the DNS, hence requiring an additional boundary condition on the temperature.

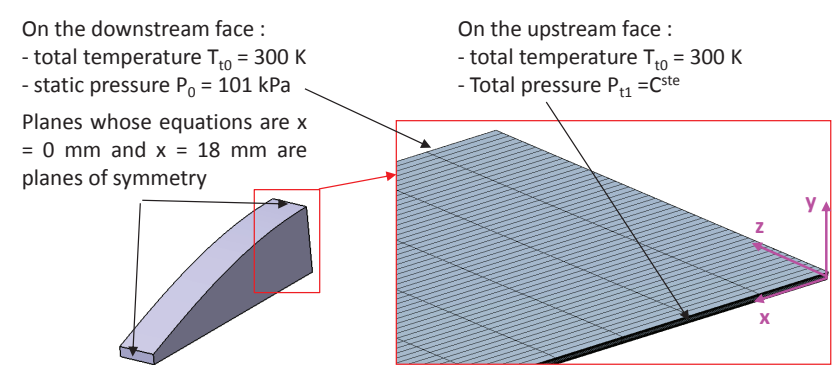

Figure 3. Boundary conditions for the numerical model. 
Adiabatic conditions are considered at the boundaries $y=$ 0 (the bore surface) and at the sinusoidal face corresponding to the model ring surface. A prescribed velocity in the axial direction, $V=10 \mathrm{~ms}^{-1}$, is assumed at this surface, accounting for the piston velocity. The flow is assumed to occur in the laminar regime and the equations are those given in the gas flow model section (see equations (1) to (4)). The computation is performed using the ANSYS Fluent ${ }^{\circledR}$ software. ${ }^{33}$ A mesh convergence test was first carried out, indicating that the selected mesh was fine enough to reach this convergence.

Three simulations, corresponding to the three expected regimes identified above were carried out, using three different values of the total pressure condition, $P_{t 1}$, at the upstream face of fluid domain $(z=0)$, namely

* Case $1 P_{t 1}=P_{0}+1 k P a$

In this case, $\tau \sim 0.01$ for which the incompressible regime is expected.

* Case $2 P_{t 1}=P_{0}+20 \mathrm{kPa}$

In this configuration, $\tau \sim 0.1$ and the flow is expected to occur in the compressible creeping regime.

* Case $3 P_{t 1}=P_{0}+100 \mathrm{kPa}$

In this last case, $\tau \sim 1$ for which the expected flow regime is the compressible inertial one.

\section{Comparison of the velocity fields}

The values of the axial component of the velocity, $w$, given by the simplified models are compared to the results obtained from the DNS. The comparison is carried out in the midsection of the bore-ring interface, at $z=0.5 \mathrm{~mm}$ and in the zone where the aperture is maximum, i.e. at $x=0$ where $h=$ $20 \mu \mathrm{m}$. This comparison is evaluated through the relative percent error between the DNS results and the predictions of the three models (incompressible, compressible creeping and compressible inertial).

Results for case $1\left(P_{t 1}=P_{0}+1 k P a\right)$ are reported in Fig. 4. In this situation, $\tau \ll 1$, and the velocity field is well predicted by the incompressible flow model, the two other models yielding the same results. The $\%$ error on $w$ is less than $2 \%$ in that case.

Results on $w$ for case $2,\left(P_{t 1}=P_{0}+20 k P a\right)$ are represented in Fig. 5. In that case, compressibility effects become significant, as expected, although the compressible creeping model and the incompressible model seem equivalent in this area of the bore-ring interface. For these two models, the maximum error is $8.5 \%$. Since $\beta$ is of order 1 , inertial effects are not completely negligible. For this reason, the compressible inertial model performs a little bit better than the two other ones, the relative error on $w$ remaining below $6.8 \%$ with this model.

For case 3 , for which $P_{t 1}=P_{0}+100 k P a(\tau \sim 1)$, the results on $w$ are represented in Fig. 6. In this case, the upstream pressure is high enough to induce important inertial effects. The prediction of the velocity by the compressible inertial model is reasonable as the maximum relative percent error is about $15 \%$. As expected, the two other models badly perform, yielding up to $38 \%$ and $\sim 33 \%$ for the incompressible and compressible models, respectively.

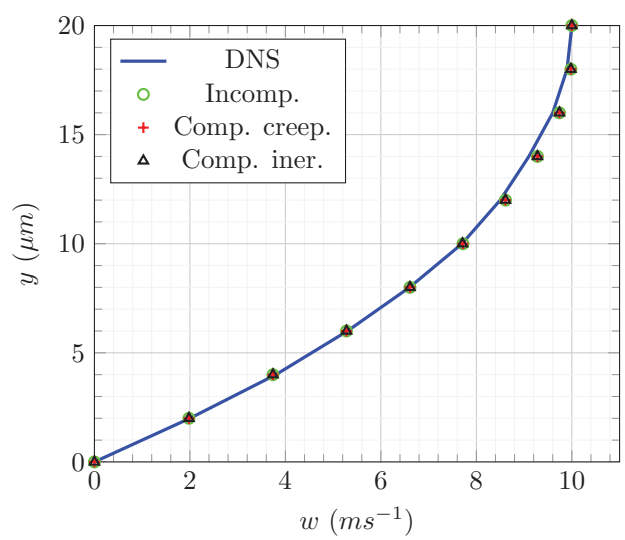

a)

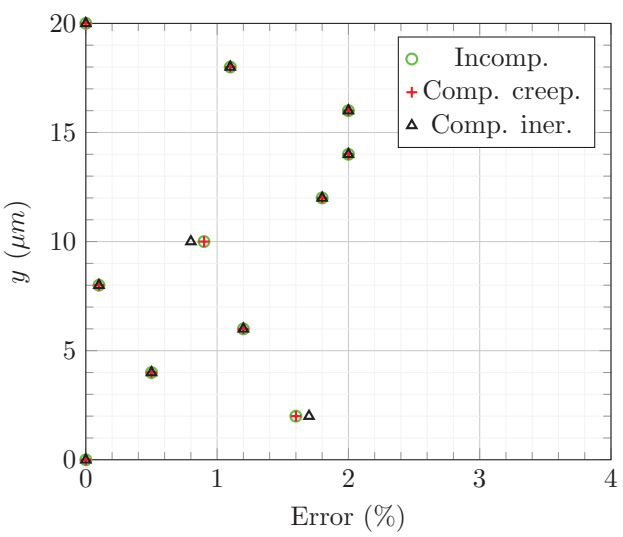

b)

Figure 4. a) Variation of the velocity axial component in the radial direction at $z=0.5 \mathrm{~mm}$. Comparison between the DNS results and the predictions obtained from the incompressible, compressible creeping and compressible inertial models. b) Relative \% error of the predictions with respect to the DNS results. Case $1(\tau \sim 0,01)$.

\section{Comparison of the pressure fields}

The axial variation of the pressure predicted by each of the simplified models is compared to the result of the DNS in the area of maximal aperture $(x=0, y=10 \mu m, h=20 \mu m)$, in the three cases under consideration.

For case $1\left(P_{t 1}=P_{0}+1 k P a\right)$, which results are shown in Fig. 7, the pressure variation is quasi linear. This is consistent with a flow in the incompressible regime. As observed on the profile of the axial component of the velocity, the models are almost equivalent. The incompressible flow model yields an excellent prediction of the pressure with less than $0.025 \%$ of error.

In case $2\left(P_{t 1}=P_{0}+20 \mathrm{kPa}\right)$, the weak compressibility effects induce a slight non linearity of the pressure profile as can be noticed in Fig. 8. In this case, the three models perform equivalently, the $\%$ error remaining smaller than $0.3 \%$ for the compressible creeping model, confirming the relevance of this simplified prediction.

For case 3 , corresponding to $P_{t 1}=P_{0}+100 k P a$, the curvature of the pressure profile due to compressibility effects is much more pronounced (see Fig. 9). The prediction from the compressible inertial model is excellent, leading to a $\%$ error of less than $1.4 \%$, while, for the incompressible and compressible creeping models it reaches $8.7 \%$ and $2.8 \%$, 


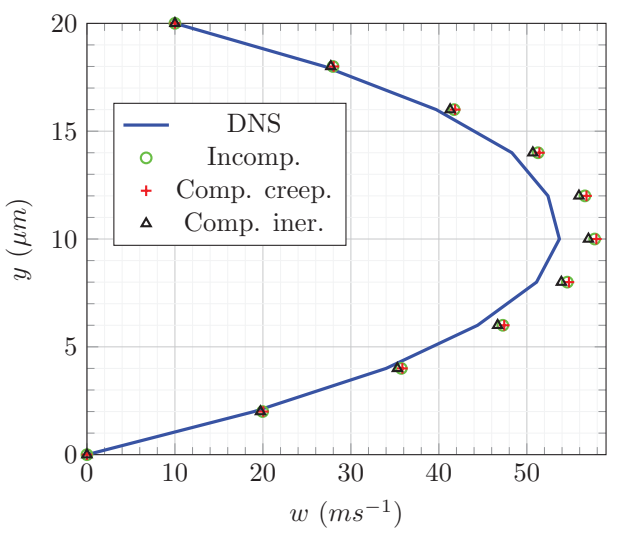

a)

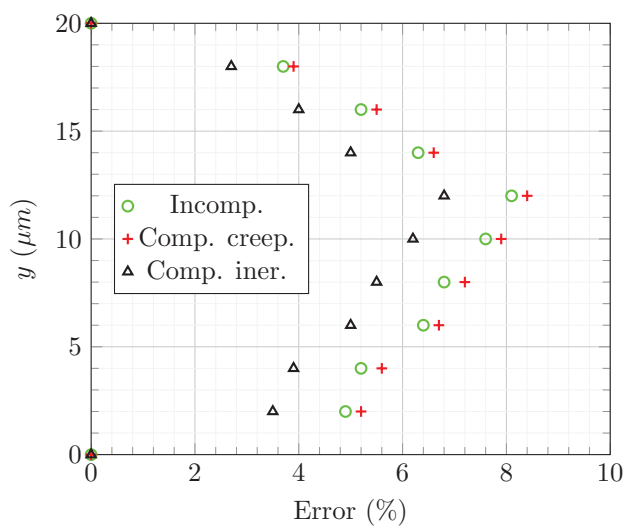

b)

Figure 5. a) Variation of the velocity axial component in the radial direction at $z=0.5 \mathrm{~mm}$. Comparison between the DNS results and the predictions obtained from the incompressible, compressible creeping and compressible inertial models. b) Relative \% error of the predictions with respect to the DNS results. $\underline{\text { Case } 2}(\tau \sim 0,1)$.

respectively. The poor performance of the incompressible model is partly due to density variations. Additionally, there is also an effect induced by the boundary conditions at the upstream face. Indeed, the static pressure, $P_{1}(x)$, decreases when the axial component of the velocity increases. Since the velocity predicted with the incompressible model is overestimated, an error is induced on the total pressure, $P_{t 1}$, and the static pressure, $P_{1}$, at the upstream face is also overestimated. As a result, the model including inertia is the most accurate one.

\section{Comparison of the mass flow rates}

Mass flow rate is the main variable of interest as it directly impacts engine performance. In Fig. 10, the computed flow rates through the sinusoidal aperture field (over one period) are represented versus the total pressure difference between the upstream and downstream faces of the contact.

When $\tau \ll 1$, which is equivalent in this case to $\Delta P<$ $100 k P a$, the simplified creeping models provide reasonable estimates of the mass flow rate. For $\Delta P=50 \mathrm{kPa}$, the incompressible model yields a relative error of about $31 \%$, the compressible creeping model performing better with less than $15 \%$ of relative error.

The discrepancy between the flow rates computed from the inertial simplified model and those obtained from DNS

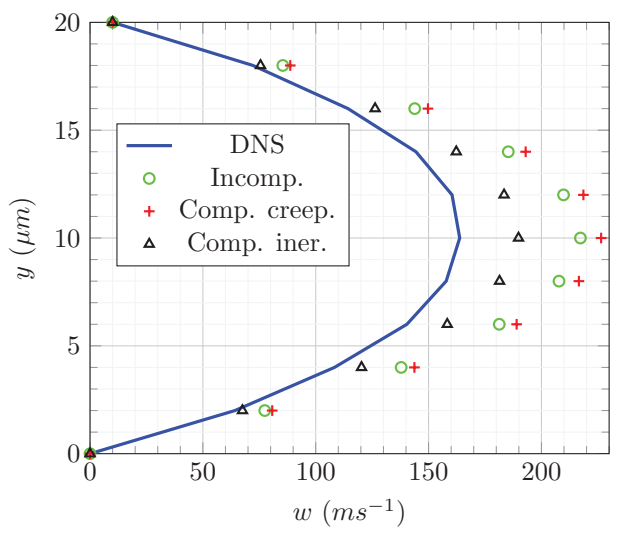

a)

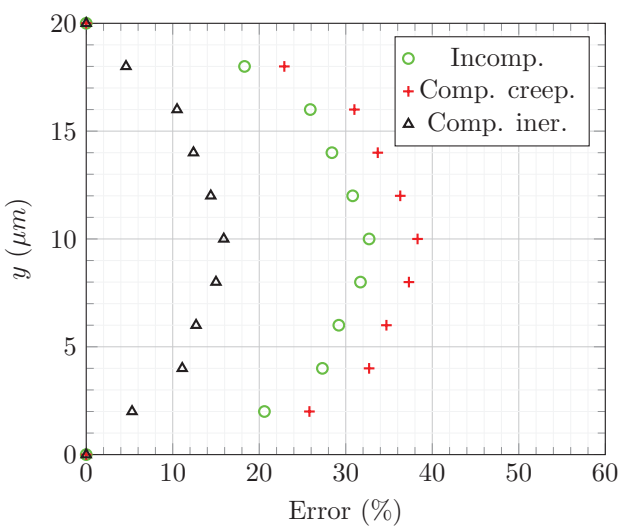

b)

Figure 6. a) Variation of the velocity axial component in the radial direction at $z=0.5 \mathrm{~mm}$. Comparison between the DNS results and the predictions obtained from the incompressible, compressible creeping and compressible inertial models. b) Relative \% error of the predictions with respect to the DNS results. $\underline{\text { Case } 3}(\tau \sim 1)$.

remains less than $12 \%$ and does not increase while increasing the pressure difference $\Delta P$. This clearly validates this predictive model making this tool an operational one to estimate the flow rate through the aperture field when $\tau$ is of order 1.

In practice, an operational use of the models derived above may be considered as to select the configuration (ring in a pack, position along the bore) which gives the largest flow rate for a secure prediction. Similarly, should the value of $\tau$ be in between the limits of applicability of one model or another (for instance $\tau=0.5$ ), so that it may be difficult to select the appropriate model a priori, the recommendation would be to compute the flow with the two models corresponding to the two closet ranges of $\tau$ and select the one which would predict the largest flow rate.

In the case of an IC engine, the use of the above models also requires a careful attention regarding the different operating cycle periods. However, a thorough analysis taking into account these features which may be very different from one case to another, is beyond the scope of the present work and is left for a more detailed future work. 


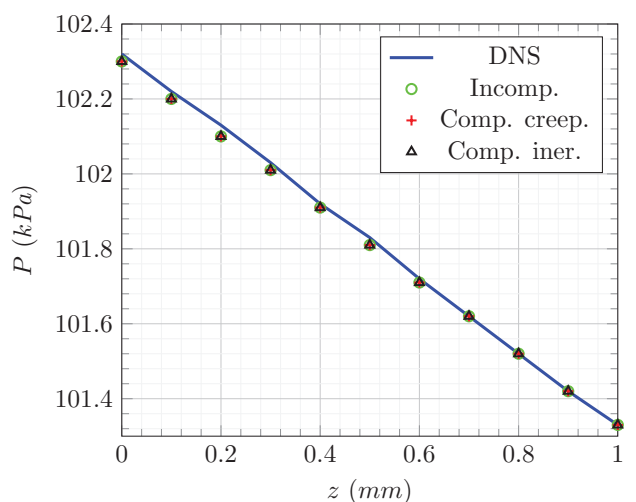

a)

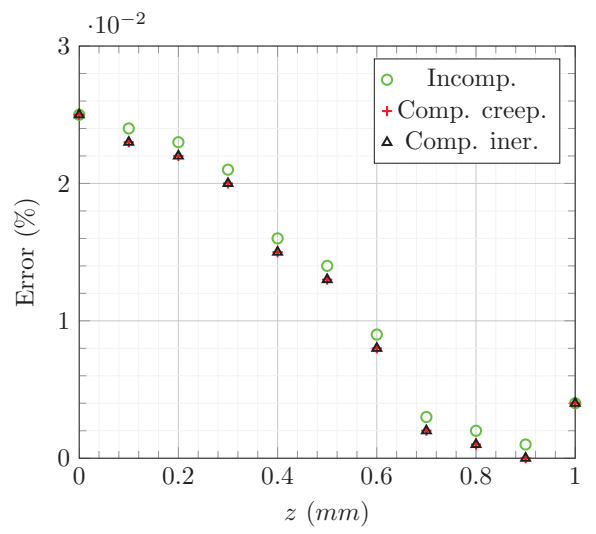

b)

Figure 7. a) Pressure variation in the axial direction at $x=0$, $y=10 \mu m,(h=20 \mu m)$. a) Comparison of the DNS results with the predictions obtained from the incompressible, compreesible creeping and compressible inertial models. b) Relative \% error of the predictions with respect to the DNS results. Case $1(\tau \sim 0,01)$.

\section{Discussion and conclusion}

In this study, the gas blow-by through a bore-ring contact, as might be encountere in reciprocating machines, is carefully analyzed with the purpose of deriving simplified accurate operational models, avoiding direct numerical simulations that would be hardly feasible in practice. Three simplified quasi analytical models to estimate the gas flow-rate through the aperture field of the contact are derived. The analysis shows that sealing of the clearance between a piston-ring and a bore is a quasi steady problem as non-stationary terms in balance equations governing the flow can be reasonably neglected. The unsteady character of the flow remains only in the boundary condition at the gas-ring interface that moves at a velocity $V$ (which is time dependent over the piston stroke in real situations) and in the change of the aperture field of the bore-ring contact along the piston stroke.

Predictions of the gas axial velocity and pressure as well as of the gas mass flow-rates from these models are compared to those obtained from direct numerical simulation (DNS) of the flow on a model aperture field of realistic dimensions. This successful comparison confirms the validity of these predictive models that are of simple and rapid use and only require the knowledge of the aperture field. They allow an accurate estimation of the blow-by through a piston-ring interface that could hardly be achieved from DNS otherwise.

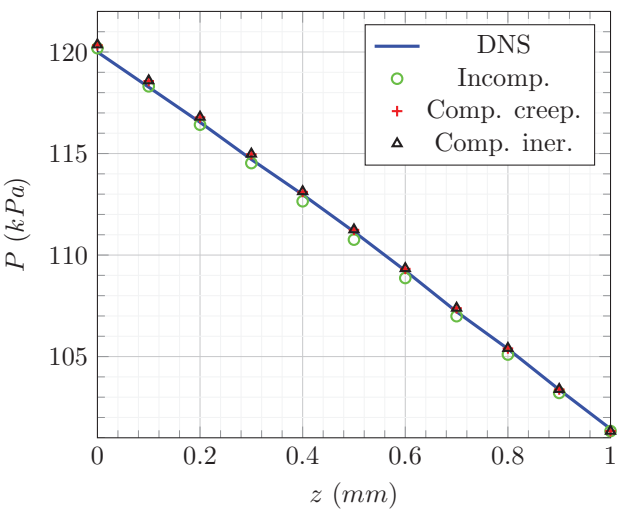

a)

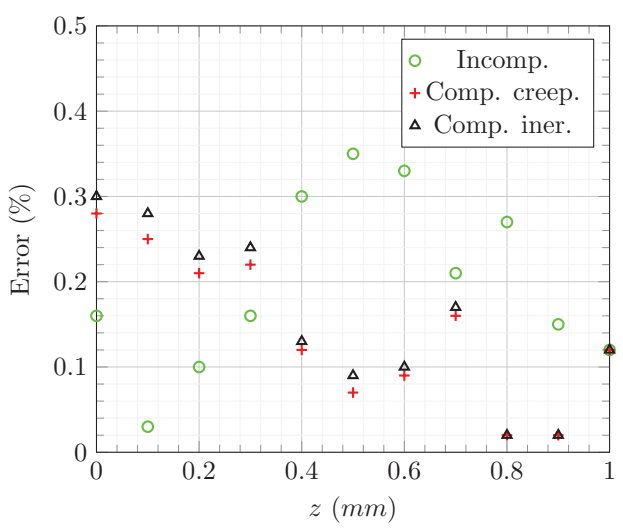

b)

Figure 8. a) Pressure variation in the axial direction at $x=0$, $y=10 \mu m,(h=20 \mu m)$. a) Comparison of the DNS results with the predictions obtained from the incompressible, compreesible creeping and compressible inertial models. b) Relative \% error of the predictions with respect to the DNS results. Case $2(\tau \sim 0,1)$.

They could be advantageously implemented in discrete time models for the motion of a piston ring pack, allowing to quantify the drop in the compression ratio of a reciprocating machine.

The derivation of the models reported in this work was made within the framework of simplifying assumptions including $i$ ) the ideal gas law; ii) constant thermophysical parameters (dynamic viscosity, heat capacity at constant pressure and thermal conductivity); iii) no oil film present; $i v)$ values of $\tau$ up to 1 . These assumptions were considered for the sake of simplicity with the purpose of proposing a methodology. Models may be further enriched by reconsidering these hypotheses. As a first approach, the oil film may be taken into account by first distributing the oil within the asperities compatible with the equilibrium of the oil-phase subject to viscous shear forces due to gas flow and capillary forces. This would result in a modified aperture field which could then be used to estimate the gas blowby using the models proposed here. Regarding values of $\tau$ larger than 1 , which are encountered in IC engine, in particular for the top ring, the results reported in Fig. 10 suggest that the compressible inertial flow model allows to accurately predict the gas mass flow rate beyond this limit of $\tau$, the error with respect to the value obtained from DNS remaining around $10 \%$. Nevertheless, a more thorough analysis is necessary to confirm this. The dependence of the 


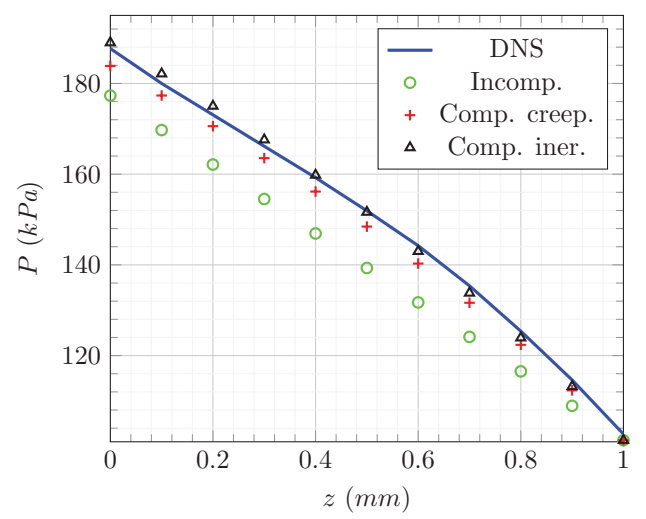

a)

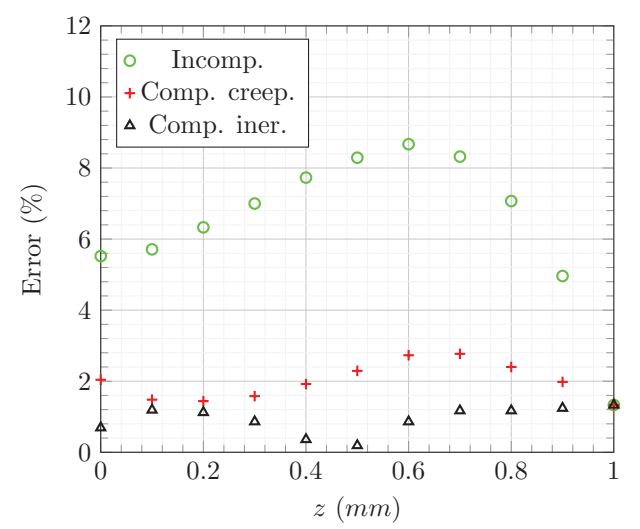

b)

Figure 9. a) Pressure variation in the axial direction at $x=0$, $y=10 \mu m,(h=20 \mu m)$. a) Comparison of the DNS results with the predictions obtained from the incompressible, compreesible creeping and compressible inertial models. b) Relative \% error of the predictions with respect to the DNS results. $\underline{\text { Case } 3}(\tau \sim 1)$.

thermophysical parameters upon the temperature and fuelto-air ratio in the case of an IC engine also deserves some additional work which was beyond the scope of the present analysis. As a final remark, it shall be emphasized that the models reported in this work are particularly relevant in many applications in turbo machines where rings may be static and operates in the absence of oil.

The methodology (and the models) proposed in this work can be of major interest for the diagnostic and/or design of more efficient bore-piston assembly making use of ring packs. A similar approach could be employed for many other similar problems of gas flow in a fracture.

\section{Appendix: Solutions in terms of orders of magnitude}

In this appendix, details on how to derive the scales for the velocity components, pressure, temperature and density are provided. This is achieved by solving the equations in terms of orders of magnitude, as is usually done, for instance, in the method of matched asymptotic expansions. ${ }^{32}$

The axial projection of the Navier-Stokes equation (2), written in its dimensionless form, takes the following

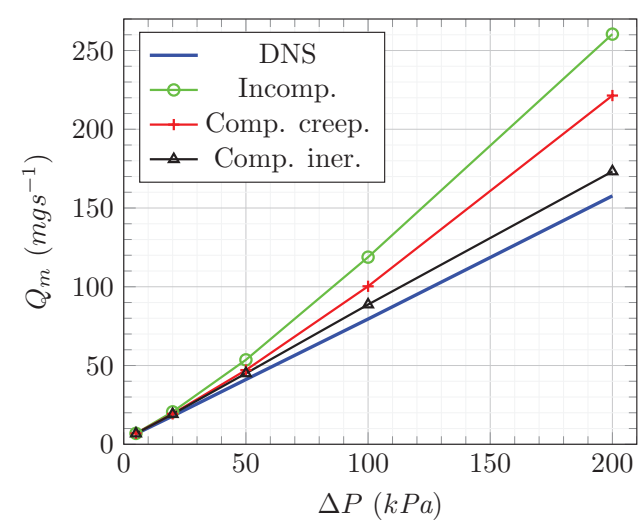

a)

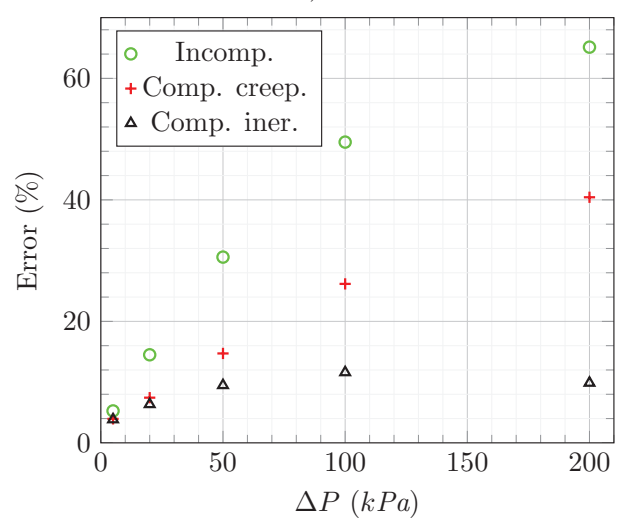

b)

Figure 10. a) Mass flow rate through one period of the sinusoidal aperture field. Comparison between the results obtained from DNS and those predicted by the incompressible, compressible creeping and compressible inertial models. b) Relative \% error of the predictions relative to the DNS results.

expression

$$
\begin{aligned}
& \frac{1}{r T_{0}} \frac{\frac{P_{0}}{\Delta P}+P^{*}}{1+\frac{\Delta T}{T_{0}} T^{*}} l_{0} w_{0} \\
& \left(\frac{1}{t_{0}} \frac{\partial w^{*}}{\partial t^{*}}+\frac{u_{0}}{L_{0}} u^{*} \frac{\partial w^{*}}{\partial x^{*}}+\frac{v_{0}}{h_{0}} v^{*} \frac{\partial w^{*}}{\partial y^{*}}+\frac{w_{0}}{l_{0}} w^{*} \frac{\partial w^{*}}{\partial z^{*}}\right) \\
& =-\frac{\partial P^{*}}{\partial z^{*}}+\frac{\mu l_{0} w_{0}}{h_{0}^{2} \Delta P}\left(\epsilon^{2} \frac{\partial^{2} w^{*}}{\partial x^{* 2}}+\frac{\partial^{2} w^{*}}{\partial y^{* 2}}+\delta^{2} \frac{\partial^{2} w^{*}}{\partial z^{* 2}}\right) \\
& +\frac{1}{3} \frac{\mu}{\Delta P}\left(\frac{u_{0}}{L_{0}} \frac{\partial^{2} u^{*}}{\partial z^{*} \partial x^{*}}+\frac{v_{0}}{h_{0}} \frac{\partial^{2} v^{*}}{\partial z^{*} \partial y^{*}}+\frac{w_{0}}{l_{0}} \frac{\partial^{2} w^{*}}{\partial z^{* 2}}\right)
\end{aligned}
$$

All the dimensionless variables and their derivatives are of order 1. In particular, the first term on the right hand side of the above equation, i.e. the axial pressure gradient, is of order 1. In order to keep the second term on the right hand side, i.e. the first viscous term, in the description of the physics, the following scaling must be adopted

$$
\frac{\mu l_{0} w_{0}}{h_{0}^{2} \Delta P} \sim 1
$$

This leads to the scale for $w$ given by

$$
w_{0} \sim \frac{h_{0}^{2} \Delta P}{\mu l_{0}}
$$


Similarly, the orthoradial projection of the Navier-Stokes equation (2), written in its dimensionless form, leads to

$$
\begin{aligned}
& \frac{1}{r T_{0}} \frac{\frac{P_{0}}{\Delta P}+P^{*}}{1+\frac{\Delta T}{T_{0}} T^{*}} L_{0} \\
& \left(\frac{u_{0}}{t_{0}} \frac{\partial u^{*}}{\partial t^{*}}+\frac{u_{0}^{2}}{L_{0}} u^{*} \frac{\partial u^{*}}{\partial x^{*}}+\frac{v_{0} u_{0}}{h_{0}} v^{*} \frac{\partial u^{*}}{\partial y^{*}}+\frac{w_{0} u_{0}}{l_{0}} w^{*} \frac{\partial u^{*}}{\partial z^{*}}\right) \\
& =-\frac{\partial P^{*}}{\partial x^{*}}+\mu \frac{L_{0} u_{0}}{\Delta P h_{0}^{2}}\left(\epsilon^{2} \frac{\partial^{2} u^{*}}{\partial x^{* 2}}+\frac{\partial^{2} u^{*}}{\partial y^{* 2}}+\delta^{2} \frac{\partial^{2} u^{*}}{\partial z^{* 2}}\right) \\
& +\frac{1}{3} \frac{\mu}{\Delta P}\left(\frac{u_{0}}{L_{0}} \frac{\partial^{2} u^{*}}{\partial x^{* 2}}+\frac{v_{0}}{h_{0}} \frac{\partial^{2} v^{*}}{\partial x^{*} \partial y^{*}}+\frac{w_{0}}{l_{0}} \frac{\partial^{2} w^{*}}{\partial x^{*} \partial z^{*}}\right)
\end{aligned}
$$

Again, keeping the second term on the right hand side in the physical description requires the following scaling

$$
\frac{\mu L_{0} u_{0}}{\Delta P h_{0}^{2}} \sim 1
$$

yielding the scale for $u$

$$
u_{0} \sim \frac{h_{0}^{2} \Delta P}{\mu L_{0}}
$$

To continue the derivation of the scales for the other variables, let the velocity components $w$ and $u$ be replaced, from this point on, by their solutions, (in terms of scales, respectively $\frac{h_{0}^{2} \Delta P}{\mu l_{0}}$ and $\left.\frac{h_{0}^{2} \Delta P}{\mu L_{0}}\right)$, in the balance equations. In its dimensionless form, the continuity equation (1) writes

$$
\begin{aligned}
& \frac{\mu l_{0}^{2}}{h_{0}^{2} \Delta P t_{0}}\left(\frac{\Delta P}{P_{0}+\Delta P P^{*}} \frac{\partial P^{*}}{\partial t^{*}}-\frac{\Delta T}{T_{0}+\Delta T T^{*}} \frac{\partial T^{*}}{\partial t^{*}}\right) \\
& +(\epsilon / \delta)^{2} \frac{\partial u^{*}}{\partial x^{*}}+\frac{\mu l_{0}^{2} v_{0}}{h_{0}^{3} \Delta P} \frac{\partial v^{*}}{\partial y^{*}}+\frac{\partial w^{*}}{\partial z^{*}} \\
& +(\epsilon / \delta)^{2} u^{*}\left(\frac{1}{\frac{P_{0}}{\Delta P}+P^{*}} \frac{\partial P^{*}}{\partial x^{*}}-\frac{1}{\frac{T_{0}}{\Delta T}+T^{*}} \frac{\partial T^{*}}{\partial x^{*}}\right) \\
& +\frac{\mu l_{0}^{2} v_{0}}{h_{0}^{3} \Delta P} v^{*}\left(\frac{1}{\frac{P_{0}}{\Delta P}+P^{*}} \frac{\partial P^{*}}{\partial y^{*}}-\frac{1}{\frac{T_{0}}{\Delta T}+T^{*}} \frac{\partial T^{*}}{\partial y^{*}}\right) \\
& +w^{*}\left(\frac{1}{\frac{P_{0}}{\Delta P}+P^{*}} \frac{\partial P^{*}}{\partial z^{*}}-\frac{1}{\frac{T_{0}}{\Delta T}+T^{*}} \frac{\partial T^{*}}{\partial z^{*}}\right)=0
\end{aligned}
$$

To satisfy the least degeneracy principle and keep the third and fifth terms on the left hand side of this equation, it is required that

$$
\frac{\mu l_{0}^{2} v_{0}}{h_{0}^{3} \Delta P} \sim 1
$$

i.e. the scale for $v$ to be given by

$$
v_{0} \sim \frac{h_{0}^{3} \Delta P}{\mu l_{0}^{2}}
$$

Let the velocity component $v$ be now replaced by its solution (in terms of scales) $\frac{h_{0}^{3} \Delta P}{\mu l_{0}^{2}}$ in the balance equations.
The energy equation (3) becomes

$$
\begin{aligned}
& \frac{h_{0}^{2}}{r \mu t_{0}} \frac{\Delta P}{\Delta T}\left[\frac{\mu^{2} l_{0}^{2}\left(c_{p}-r\right) T_{0}}{h_{0}^{4} \Delta P^{2}}\left(1+\frac{\Delta T}{T_{0}} T^{*}\right)+O\left((\epsilon / \delta)^{2}\right)\right. \\
& \left.+O\left(\delta^{2}\right)\right]\left(\frac{1}{\frac{T_{0}}{\Delta T}+T^{*}} \frac{\partial P^{*}}{\partial t^{*}}-\frac{\frac{P_{0}}{\Delta P}+P^{*}}{\left(\frac{T_{0}}{\Delta T}+T^{*}\right)^{2}} \frac{\partial T^{*}}{\partial t^{*}}\right) \\
& +\frac{h_{0}^{2}}{r \mu t_{0}} \frac{\Delta P}{\Delta T} \frac{\frac{P_{0}}{\Delta P}+P^{*}}{\left(\frac{T_{0}}{\Delta T}+T^{*}\right)} \\
& \left(\frac{\mu^{2} l_{0}^{2}\left(c_{p}-r\right) \Delta T}{h_{0}^{4} \Delta P^{2}} \frac{\partial T^{*}}{\partial t^{*}}+O\left((\epsilon / \delta)^{2}\right)+O\left(\delta^{2}\right)\right) \\
& +\frac{1+\frac{\Delta P}{P_{0}} P^{*}}{1+\frac{\Delta T}{T_{0}} T^{*}}\left[\frac{P_{0} h_{0}^{4} \Delta P}{r T_{0} \mu^{2} l_{0}^{2}} w^{*}\left(v^{*} \frac{\partial w^{*}}{\partial y^{*}}+w^{*} \frac{\partial w^{*}}{\partial z^{*}}\right)\right. \\
& \left.+\frac{P_{0} c_{p} \Delta T}{r T_{0} \Delta P}\left(v^{*} \frac{\partial T^{*}}{\partial y^{*}}+w^{*} \frac{\partial T^{*}}{\partial z^{*}}\right)\right] \\
& =\frac{\mu l_{0}^{2} \lambda \Delta T}{h_{0}^{4} \Delta P^{2}} \frac{\partial^{2} T^{*}}{\partial y^{* 2}}+\frac{\partial}{\partial y^{*}}\left(w^{*} \frac{\partial w^{*}}{\partial y^{*}}\right) \\
& +O\left((\epsilon / \delta)^{2}\right)+O\left(\epsilon^{2}\right)+O\left(\delta^{2}\right)
\end{aligned}
$$

At this point, two assumptions can be made consisting in considering that either the convection or the conduction term is of the same order of magnitude as the viscous dissipation term and this is detailed below.

\section{Convection and viscous dissipation terms are of the same order of magnitude}

With this assumption, it follows that

$$
\frac{P_{0} c_{p} \Delta T}{r T_{0} \Delta P} \sim 1
$$

This further implies that the scaling for $T$ is given by

$$
\Delta T \sim \frac{r T_{0} \Delta P}{P_{0} c_{p}}
$$

As a consequence, the energy equation (A10) can be written as

$$
\begin{aligned}
& \alpha \operatorname{Re} \delta \frac{c_{p}}{r} \frac{1}{\tau}\left[\frac{1}{\operatorname{Re} \delta}\left(\frac{c_{p}}{r}-1\right) \frac{1}{\tau}\left(1+\tau \frac{r}{c_{p}} T^{*}\right)+O\left((\epsilon / \delta)^{2}\right)\right. \\
& \left.+O\left(\delta^{2}\right)\right]\left(\frac{1}{\frac{c_{p}}{r} \frac{1}{\tau}+T^{*}} \frac{\partial P^{*}}{\partial t^{*}}-\frac{\frac{1}{\tau}+P^{*}}{\left(\frac{c_{p}}{r} \frac{1}{\tau}+T^{*}\right)^{2}} \frac{\partial T^{*}}{\partial t^{*}}\right) \\
& +\alpha \operatorname{Re} \delta \frac{c_{p}}{r} \frac{1}{\tau} \frac{\frac{1}{\tau}+P^{*}}{\left(\frac{c_{p}}{r} \frac{1}{\tau}+T^{*}\right)} \\
& \left(\frac{1}{\operatorname{Re} \delta}\left(1-\frac{r}{c_{p}}\right) \frac{\partial T^{*}}{\partial t^{*}}+O\left((\epsilon / \delta)^{2}\right)+O\left(\delta^{2}\right)\right) \\
& +\frac{1+\tau P^{*}}{1+\frac{r}{c_{p}} \tau T^{*}}\left[\operatorname{Re} \delta w^{*}\left(v^{*} \frac{\partial w^{*}}{\partial y^{*}}+w^{*} \frac{\partial w^{*}}{\partial z^{*}}\right)\right. \\
& \left.+v^{*} \frac{\partial T^{*}}{\partial y^{*}}+w^{*} \frac{\partial T^{*}}{\partial z^{*}}\right]=\frac{1}{\operatorname{Pr}} \frac{1}{\operatorname{Re} \delta} \frac{\partial^{2} T^{*}}{\partial y^{* 2}} \\
& +\frac{\partial}{\partial y^{*}}\left(w^{*} \frac{\partial w^{*}}{\partial y^{*}}\right)+O\left((\epsilon / \delta)^{2}\right)+O\left(\epsilon^{2}\right)+O\left(\delta^{2}\right)
\end{aligned}
$$

with $\tau=\frac{\Delta P}{P_{0}}, \operatorname{Re} \delta=\frac{P_{0}^{2}}{r T_{0}} \frac{h_{0}^{4}}{\mu^{2} l_{0}^{2}} \tau, \operatorname{Pr}=\frac{\mu c_{p}}{\lambda}=0.74, \frac{r}{c_{p}}=$ $0.29, \alpha=\frac{\mu l_{0}^{2}}{P_{0} t_{0} h_{0}^{2}}$. The numerical values provided here are those for air at $300 \mathrm{~K}$ and $10^{5} \mathrm{~Pa}$. 
To assess the validity of this approximation which results from matching the orders of magnitude of the convection and viscous dissipation terms, it is of interest to look for a solution to the above equation, and for the sake of simplicity, in an analytical form. This can be achieved in the creeping flow regime, when $R e \delta \ll 1$. In this regime, the energy equation (A13) becomes

$$
\begin{aligned}
O\left(\alpha \beta / \tau^{2}\right)+\frac{\partial^{2} T^{*}}{\partial y^{* 2}} & =O\left((\epsilon / \delta)^{2}\right)+O\left(\epsilon^{2}\right) \\
& +O\left(\delta^{2}\right)+O\left((\beta \tau)^{2}\right)
\end{aligned}
$$

Further assuming that $\frac{\alpha \beta}{\tau^{2}} \ll 1$, and with the boundary conditions $T(y=0)=T(y=h)=T_{0}$, the integration of equation (A14) leads to

$$
T(y)=T_{0}
$$

This last result indicates that the temperature field would be uniform in both the axial and radial directions.

To check the validity of this conclusion, the solution given by equation (A15) can be compared to the one obtained from a direct numerical simulation (DNS), with a model similar to the one presented in the section "Comparison with DNS" in the body of the article. The simulation is performed with a maximum aperture of $10 \mu \mathrm{m}$ while considering the following boundary conditions: $T_{t 1}=300 \mathrm{~K}, P_{t 1}=121 \mathrm{kPa}, P_{0}=$ $101 \mathrm{kPa}, T_{t 0}=300 \mathrm{~K}, V=10 \mathrm{~ms}^{-1}$. These values ensure $\beta=O(1), \tau=O(0.1), \alpha=O\left(10^{-4}\right)$ which means $\operatorname{Re} \delta=\beta \tau=O(0.1)$ and $\frac{\alpha \beta}{\tau^{2}}=O\left(10^{-2}\right)$, which satisfy the constraints formulated to obtain the analytical solution in equation (A15).

Results on the temperature fields obtained from this simulation are represented versus the radial coordinate $y$ for three axial positions $(z=0.1 \mathrm{~mm}, z=0.5 \mathrm{~mm}$ and $z=0.9$ $\mathrm{mm})$ at $x=1 \mathrm{~mm}$ are represented in Fig. A1. These results show that the temperature field is not rigorously uniform.

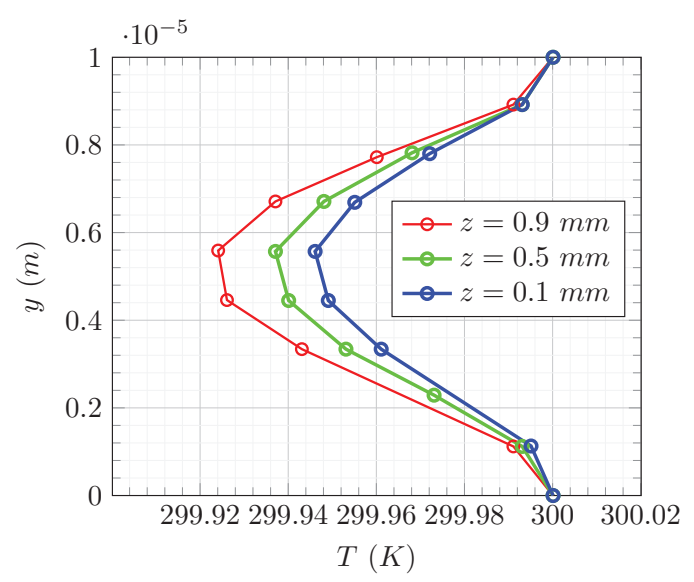

Figure A1. Gas temperature variation in the radial direction at $x=1 \mathrm{~mm}$ and at three axial positions as obtained by DNS.

However, it must be noticed that the temperature fluctuations are very small compared to the mean temperature value. In fact, these fluctuations do not exceed few hundredth of Kelvin for an average temperature close to $300 \mathrm{~K}$. This indicates that assuming the conduction and dissipation terms to be of the same orders of magnitude is acceptable and that the temperature field in the fluid domain can be reasonably considered as uniform.

Nevertheless, it is of interest to investigate the second possible choice for the solution of the energy equation (A10).

\section{Conduction and viscous dissipation terms are of the same order of magnitude}

Making this assumption leads to consider the coefficient of the convective term in equation (A10) to be of order 1

$$
\frac{\mu l_{0}^{2} \lambda \Delta T}{h_{0}^{4} \Delta P^{2}} \sim 1
$$

and this yields the scale for $T$

$$
\Delta T \sim \frac{h_{0}^{4} \Delta P^{2}}{\mu l_{0}^{2} \lambda}
$$

Under this assumption, the energy equation (A10) becomes

$$
\begin{aligned}
& \frac{\alpha}{\tau} \frac{1}{\operatorname{Pr}} \frac{c_{p}}{r}\left[\frac{1}{\operatorname{Re} \delta \tau}\left(\frac{c_{p}}{r}-1\right)\left(1+\operatorname{Re} \delta \tau \frac{r}{c_{p}} \operatorname{Pr} T^{*}\right)\right. \\
& \left.+O\left((\epsilon / \delta)^{2}\right)+O\left(\delta^{2}\right)\right] \\
& \left(\frac{1}{\frac{c_{p}}{r} \frac{1}{\operatorname{Re} \tau \tau \operatorname{Pr}}+T^{*}} \frac{\partial P^{*}}{\partial t^{*}}-\frac{\frac{1}{\tau}+P^{*}}{\left(\frac{c_{p}}{r} \frac{1}{\operatorname{Re} \delta \tau \operatorname{Pr}}+T^{*}\right)^{2}} \frac{\partial T^{*}}{\partial t^{*}}\right) \\
& +\frac{\alpha}{\tau} \frac{1}{\operatorname{Pr}} \frac{c_{p}}{r} \frac{\frac{1}{\tau}+P^{*}}{r} \frac{1}{\operatorname{Re} \delta P_{r}}+T^{*} \\
& \left(\operatorname{Pr}\left(1-\frac{r}{c_{p}}\right) \frac{\partial T^{*}}{\partial t^{*}}+O\left((\epsilon / \delta)^{2}\right)+O\left(\delta^{2}\right)\right) \\
& +\frac{1+\tau P^{*}}{1+\operatorname{Re} \delta \frac{\mu r}{\lambda} \tau T^{*}} \operatorname{Re} \delta\left[w^{*}\left(v^{*} \frac{\partial w^{*}}{\partial y^{*}}+w^{*} \frac{\partial w^{*}}{\partial z^{*}}\right)\right. \\
& \left.+\operatorname{Pr}\left(v^{*} \frac{\partial T^{*}}{\partial y^{*}}+w^{*} \frac{\partial T^{*}}{\partial z^{*}}\right)\right]=\frac{\partial^{2} T^{*}}{\partial y^{* 2}} \\
& +\frac{\partial}{\partial y^{*}}\left(w^{*} \frac{\partial w^{*}}{\partial y^{*}}\right)+O\left((\epsilon / \delta)^{2}\right)+O\left(\epsilon^{2}\right)+O\left(\delta^{2}\right)
\end{aligned}
$$

with $\tau=\frac{\Delta P}{P_{0}}, \operatorname{Re} \delta=\frac{P_{0}^{2}}{r T_{0}} \frac{h_{0}^{4}}{\mu^{2} l_{0}^{2}} \tau, \operatorname{Pr}=\frac{\mu c_{p}}{\lambda}=0.74, \frac{\mu r}{\lambda}=$ 0.2 . Again, the numerical values provided here are those for air at $300 \mathrm{~K}$ and $10^{5} \mathrm{~Pa}$

As in the previous case, it is of interest to compare the solution of the above equation (in the case where it can be solved analytically, i.e. in the creeping flow regime) with the result of the DNS presented above. Considering again $\operatorname{Re} \delta \ll 1$ ), the energy equation (A18) takes the following form

$$
\begin{aligned}
O\left(\frac{\alpha}{\beta \tau}\right)+\frac{\partial^{2} T^{*}}{\partial y^{* 2}} & =-\frac{\partial}{\partial y^{*}}\left(w^{*} \frac{\partial w^{*}}{\partial y^{*}}\right) \\
& +O\left((\epsilon / \delta)^{2}\right)+O\left(\epsilon^{2}\right)+O\left(\delta^{2}\right)
\end{aligned}
$$

If $\frac{\alpha}{\beta \tau} \ll 1$ (this is the case in the DNS to which this present solution will be compared as $\frac{\alpha}{\beta \tau}=O\left(10^{-3}\right)$ ) and using the boundary conditions $T(y=0)=T(y=h)=T_{0}$, the 
integration of this last equation leads to

$$
\begin{aligned}
& T(x, y, z)=\frac{\mu}{\lambda} K\left(-\frac{1}{2} K y^{2}+\frac{1}{3}\left(3 h(x) K-\frac{V}{h(x)}\right) y\right. \\
& \left.+\frac{1}{2}\left(V-K h^{2}(x)\right)\right) y^{2}-\frac{K \mu V h(x)}{6 \lambda} y+T_{0}
\end{aligned}
$$

with

$$
K=\frac{P_{0}^{2}-P_{1}^{2}(x)}{4 \mu l\left(\frac{P_{0}^{2}-P_{1}^{2}(x)}{l} z+P_{1}^{2}(x)\right)^{1 / 2}}
$$

This analytical solution can now be compared to the solution obtained from DNS as reported in Fig. A2. As can be observed on this figure, the temperature fluctuations are well captured by the approximation given in equation (A20), both in the axial and radial directions although these fluctuations are very small compared to the characteristic value of the temperature.

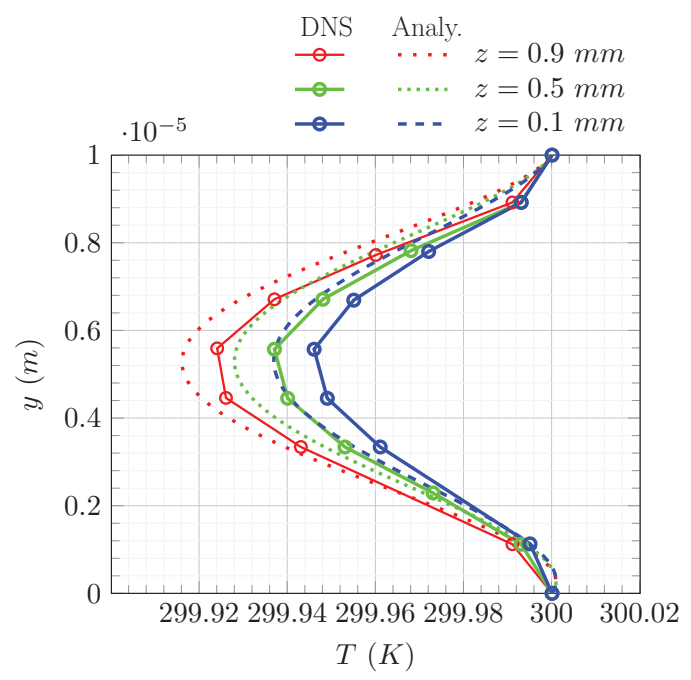

Figure A2. Gas temperature variation in the radial direction at $x=1 \mathrm{~mm}$ and at three axial positions. Comparison of the DNS results with the analytical predictions obtained from equation (A20).

To summarize, the determination of $\Delta T$ can be made from either one of the two possible assumptions investigated above. The analysis shows that conduction, convection and viscous dissipation terms are all of the same order of magnitude. However, considering that conduction and viscous dissipation are of the same order of magnitude leads to a more precise description of the temperature field. For this reason, this latter choice is kept and the scale for $T$ is that given in equation (A17).

Finally, the scale for density can be determined from the gas equation of state (4)

$$
\frac{\Delta \rho}{\rho_{0}}=\frac{1}{\rho}\left(\frac{1+\tau P^{*}}{1+\frac{\Delta T}{T_{0}} T^{*}}-1\right)
$$

i.e.

$$
\Delta \rho \sim \rho_{0}\left(\frac{1+\tau}{1+\operatorname{Re} \delta \tau \frac{\mu r}{\lambda}}-1\right)
$$

with $\tau=\frac{\Delta P}{P_{0}}, \operatorname{Re} \delta=\frac{P_{0}^{2}}{r T_{0}} \frac{h_{0}^{4}}{\mu^{2} l_{0}^{2}} \tau, \rho_{0}=\frac{P_{0}}{r T_{0}}, \frac{\mu r}{\lambda}=0.2$.
To conclude, the solution of the balance equations in terms of orders of magnitude leads to the scales for the variables $u$, $v, w, T$ and $\rho$ respectively given by

$$
\begin{gathered}
u_{0} \sim \frac{h_{0}^{2} \Delta P}{\mu L_{0}} \\
v_{0} \sim \frac{h_{0}^{3} \Delta P}{\mu l_{0}^{2}} \\
w_{0} \sim \frac{h_{0}^{2} \Delta P}{\mu l_{0}} \\
\Delta T \sim \frac{h_{0}^{4} \Delta P^{2}}{\mu l_{0}^{2} \lambda} \\
\Delta \rho \sim \rho_{0}\left(\frac{1+\tau}{1+\operatorname{Re} \delta \tau \frac{\mu r}{\lambda}}-1\right)
\end{gathered}
$$

\section{Acknowledgements}

Part of this work was carried out in the framework of the ANR project ANR-11-RSNR-0012-01, considering a problem sharing many features with the one envisaged here.

\section{References}

1. Delprete C, Selmani E, Bisha A. Gas escape to crankcase: impact of system parameters on sealing behavior of a piston cylinder ring pack. International Journal of Energy and Environmental Engineering volume. 2019;10:207-220.

2. Ting LL, Mayer JE. Piston ring lubrication and cylinder bore wear analysis. Part II-theory verification. ASME J Lubric Technol. 1974;96:258-266.

3. Brombolich LJ. Effect of cylinder distortions and piston ring design on oil consumption and friction losses in automobile engines. DOE report, No. ANL/TPRR/89/1; 1989.

4. Ma M, Sherrington I, Smith EH, Grice N. Development of a detailed model for piston-ring lubrication in IC engines with circular and non-circular cylinder bores. Tribol Int. 1997;30:779-788.

5. Tian T, Noordzij L, Wong VW, Heywood JB. Modeling piston ring dynamics, blow-by, and ring-twist effects. J Eng Gas Turbines Power. 1998;120:843-854.

6. Wannatong K, Chanchaona S, Sanitjai S. Simulation algorithm for piston ring dynamics. Simulation Modelling Practice and Theory. 2008;16:127-146.

7. Marie C, Lasseux D, Zahouani H, Sainsot P. An integrated approach to characterize liquid leakage through metal contact seal. European Journal Mech and Env Eng. 2003;48:81-86.

8. Marie C, Lasseux D. Experimental leak-rate measurement through a static metal seal. J Fluids Eng. 2007;129:799-805.

9. Bottiglione F, Carbone G, Mangialardi L, Mantriota G. Leakage mechanism in flat seals. J Appl Phys. 2009;106:104902.

10. Vallet C, Lasseux D, Sainsot P, Zahouani H. Real versus synthesized fractal surfaces: contact mechanics and transport properties. Tribol Int. 2009;42:250-259.

11. Vallet C, Lasseux D, Sainsot P, Zahouani H. Sampling effect on contact and transport properties between fractal surfaces. Tribol Int. 2009;42:1132-1145.

12. Ledoux Y, Lasseux D, Favrelière H, Samper S, Grandjean J. On the dependence of static flat seal efficiency to surface defects. Int J Press Vessels Pip. 2011;88:518-529. 
13. Bourniquel J, Lasseux D, Rit JF. Prediction and measurement of sealing properties of joints between wavy metal surfaces. J Pressure Vessel Technol. 2018;140:051203.

14. Moore SL, Hamilton GM. The starved lubrication of piston rings in a diesel engine. J Mech Engrg Sci. 1978;20:345-352.

15. Richardson DE, Borman GL. Theoretical and experimental investigations of oil films for application to piston ring lubrication. In: SAE Technical Paper. SAE International; 1992.

16. Grice N, Sherrington I. An experimental investigation into the lubrication of piston rings in an internal combustion engineoil film thickness trends, film stability and cavitation. In: SAE Technical Paper. SAE International; 1993. .

17. Tian T, Rabute R, Wong V, Heywoo JB. Effects of pistonring dynamics on the ring/groove wear and oil consumption in a diesel engine. SAE Int J Engines SAE International. 1997;106:1195-1207.

18. Tomanik E, Nigro F. Piston ring pack and cylinder wear modeling. SAE Int $\mathrm{J}$ Engines SAE International. 2001;110:585-595.

19. Harigaya Y, Suzuki M, Takiguchi M. Analysis of oil film thickness on a piston ring of diesel engine: effect of oil film temperature. J Eng Gas Turbines Power. 2003;125:596-603.

20. Green SJ, Hayward WAC, Tyrrell RJ. Analysis of bore distortion and design for low oil consumption. In: Autotech 1989; 1989. p. 132-138.

21. Schneider E, Blossfeld D, Lechman D, Hill R. Effect of cylinder bore out-of-roundness on piston ring rotation and engine oil consumption. SAE Int J Engines SAE International. 1993;102:1048-1069.

22. Delprete C, Razavykia A. Piston dynamics, lubrication and tribological performance evaluation: A review. Int J Engine Res. 2018;21:725-741.

23. Alshwawra A, Pasligh H, Hansen H, Dinkelacker F. Increasing the roundness of deformed cylinder liner in internal combustion engines by using a non-circular liner profile. Int $\mathrm{J}$ Engine Res. 2019:1-8.

24. Syed D, Wani MF. Tribological behavior of chrome-deposited SAE9254 grade steel top compression piston ring under lubrication starvation and mild extreme pressure lubrication. Int J Engine Res. 2019:1-16.

25. Li R, Meng X, Li W, Dong J. A new comprehensive tribo-dynamic analysis for lubricated translational joints in low-speed two-stroke marine engines. Int $\mathrm{J}$ Engine Res. 2019;21:1336-1361.

26. Zaouter T, Lasseux D, Prat M. Determination of the transmissivity of a heterogeneous anisotropic fracture in slip flow conditions. Phys Rev E. 2019;10:033115.

27. Torregrosa AJ, Broatch A, Margot X, Gomez-Soriano J. Understanding the unsteady pressure field inside combustion chambers of compression-ignited engines using a computational fluid dynamics approach. Int J Engine Res. 2018;21:1273-1285.

28. Gintsburg BJ. Splitless-type piston rings. Russian Engineering Journal. 1968;48:37-40.

29. Dunaevsky VV. Analysis of distortions of cylinders and conformability of piston rings. Tribol Trans. 1990;33:33-40.

30. Tomanik E. Piston Ring Conformability in a Distorted Bore. In: SAE Technical Paper. SAE International; 1996. .

31. Dunaevsky V, Rudzitis J. Clarification of a semi-empirical approach in piston/cylinder bore conformability prediction. J
Tribol. 2007;129:430-435.

32. Van Dyke M. Perturbation methods in fluid mechanics. Stanford: The Parabolic Press; 1975.

33. ANSYS Fluent User's Guide, Release 15.0; 2013. 


\section{Nomenclature}

c piston stroke, $m$

$c_{p} \quad$ heat capacity of the gas, $c_{p}=1006 \mathrm{JK}^{-1} \mathrm{~kg}^{-1}$ for air at $300 \mathrm{~K}$ and $10^{5} \mathrm{~Pa}$

$h \quad$ aperture, $m$

$h_{0} \quad$ typical aperture, $m$

$h_{\text {min }} \quad$ minimal aperture, $m$

$h_{\max }$ maximal aperture, $m$

$H \quad$ gas enthalpy, $J$

$l_{0} \quad$ typical width of a ring, $m$

$l \quad$ ring width, $m$

$L_{0} \quad$ typical distance between two contact points, $m$

$\overline{M_{1}}$ flow Mach number at the upstream face of the bore-ring interface

$P \quad$ gas pressure, $\mathrm{Pa}$

$P_{1} \quad$ pressure at the upstream face of the bore-ring interface, $\mathrm{Pa}$

$P_{0} \quad$ pressure at the downstream side of the ring, $P a$

$\mathrm{Pr} \quad$ Prandtl number

$P_{t 1} \quad$ total pressure at the upstream side of the ring, $P a$

$r \quad$ ideal gas constant, $r=287 \mathrm{JK}^{-1} \mathrm{~kg}^{-1}$ for air

Re Reynolds number

$t_{0} \quad$ stroke period, $s$

$T$ gas temperature, $K$

$T_{0} \quad$ temperature at the downstream side of the ring, K

$T_{t 1} \quad$ total temperature at the upstream side of the ring, $K$

$T_{t 0} \quad$ total temperature at the downstream side of the ring, $K$

$u \quad$ velocity component in the $x$-direction, $m s^{-1}$

$u_{0} \quad$ scale of the velocity component in the $x$-direction, $m s^{-1}$

u velocity vector, $m s^{-1}$

$v \quad$ velocity component in the $y$-direction, $\mathrm{ms}^{-1}$

$v_{0} \quad$ scale of the velocity component in the $y$-direction, $m s^{-1}$

$V \quad$ piston velocity, $m s^{-1}$

$w \quad$ velocity component in the $z$-direction, $m s^{-1}$

$w_{0} \quad$ scale of the velocity component in the $z$-direction, $m s^{-1}$

$\overline{w_{1}} \quad$ average velocity at the upstream face of the borering interface, $m s^{-1}$

\section{Greek symbols}

$\Delta P \quad$ total pressure difference between the upstream and downstream sides of the ring, $P a$

$\Delta T \quad$ scale of gas temperature, $K$

$\Delta \rho \quad$ scale of gas density, $\mathrm{kgm}^{-3}$

$\gamma \quad$ adiabatic index

$\lambda \quad$ thermal conductivity of the gas, $\lambda=2.6210^{-2}$ $W m^{-1} K^{-1}$ for air at $300 \mathrm{~K}$ and $10^{5} \mathrm{~Pa}$

$\mu \quad$ gas viscosity, $\mu=1.810^{-5}$ Pas for air at $300 \mathrm{~K}$ and $10^{5} \mathrm{~Pa}$

$\rho \quad$ gas density, $\mathrm{kgm}^{-3}$

$\rho_{0} \quad$ gas density at the downstream side of the ring, $\mathrm{kgm}^{-3}$

$\boldsymbol{\sigma} \quad$ viscous stress tensor, $\boldsymbol{\sigma}=\mu\left(\nabla \mathbf{u}+\nabla \mathbf{u}^{T}\right)$, $N m^{-2}$ 